\title{
UคPU UЪSกษ3U乙
}

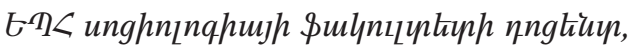

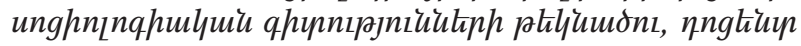

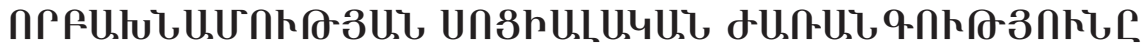

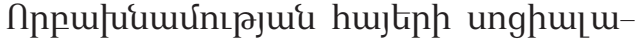

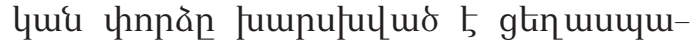

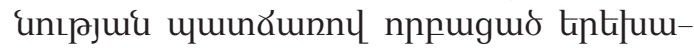
utiph huiuntiu qqujulyuu nulquintưutph nı npuiuhg plunn ununtignıưutiph htiuph

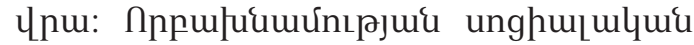

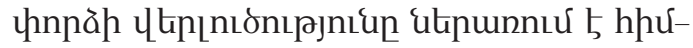

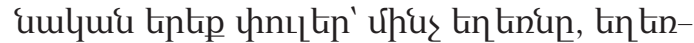

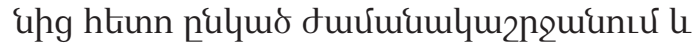
Cujuuunuip lunphpnujhu unuphutiphi:

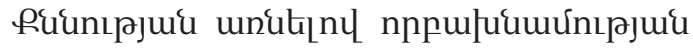
unghu[uluuu unnpăh hupnıun dunứ-

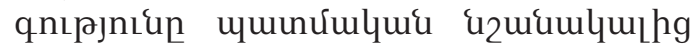

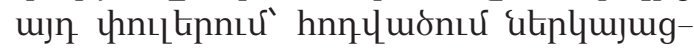

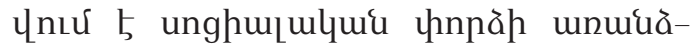

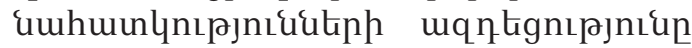

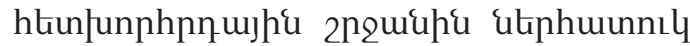

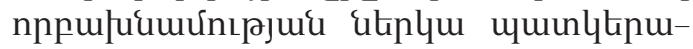

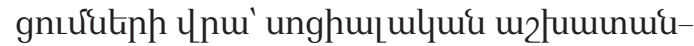

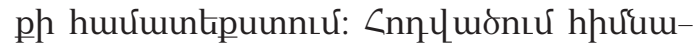

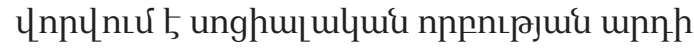

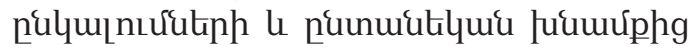

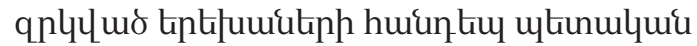

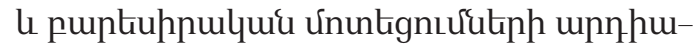

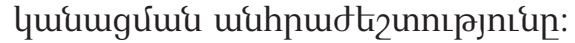

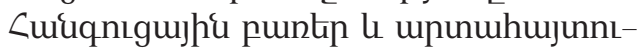
pjnikutip. npp, nppupuuuu qnpornitutns-

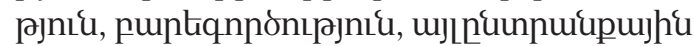

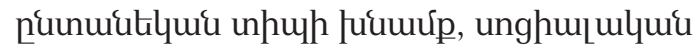

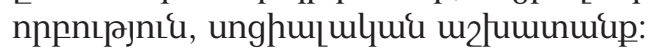

«nıph2h úuuhu hnqu[nı» quinuhu-

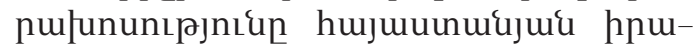

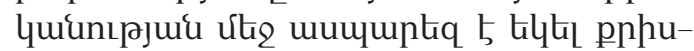

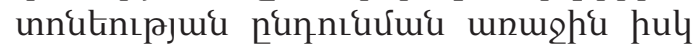

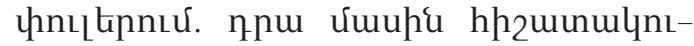

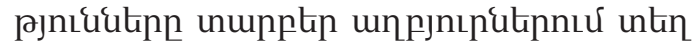

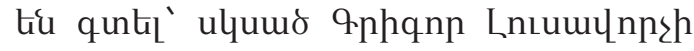

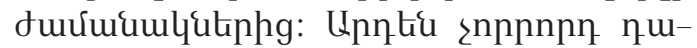

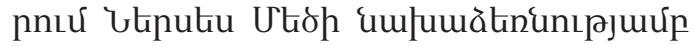

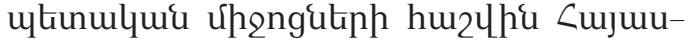
unuunsu pugltighu puquimphy punt-

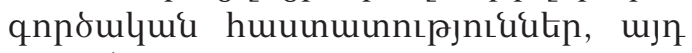
punıu' «ujptungputpp», npnup umluu-

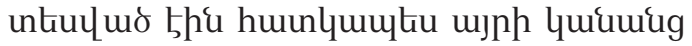

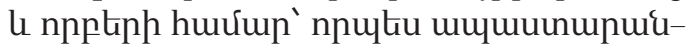
uten, npunten upuiup unumuntu thu wu-

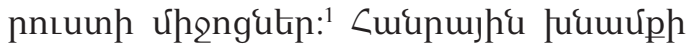

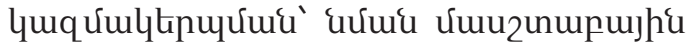
qnponupugn unpưưpumunptí unue pt-

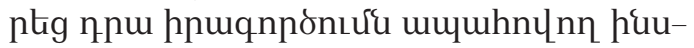
unpunnump umhuiug, huuunpunntu, nnp quinumupulunumlume huर्umumunum-

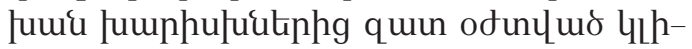
up utipphú punàn hupumlyuqưulthu-

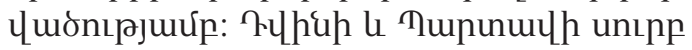

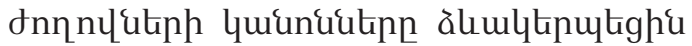
ujn ưhuugn" «... puptiqnpoulumu huuunuunıpjntưtiph hnquunupnıpjnı-

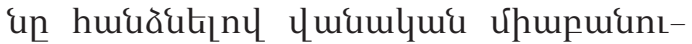

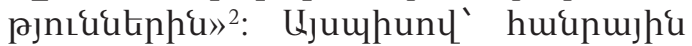

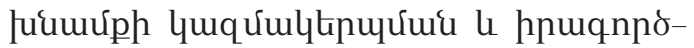

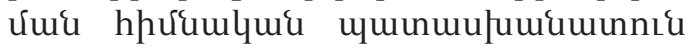

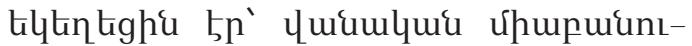

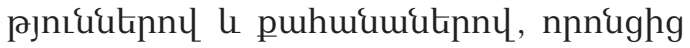
jnıpupuussnıpu hp hưắuunnıpjư hu-

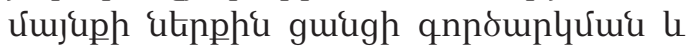

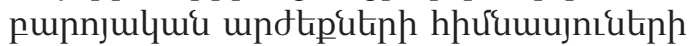

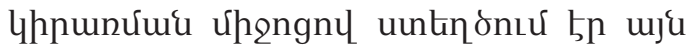
pnı\$tpujhu \$nup, nnny pnıı 25p unulhu

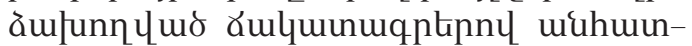
utph, huunluwutu npptiph útynıuugnt-

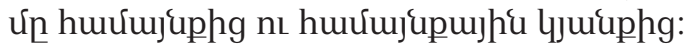

nppkph huintiu quqúultipuluo

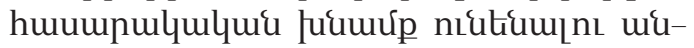

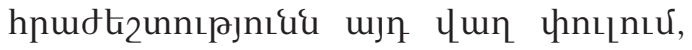

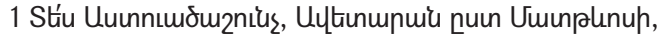

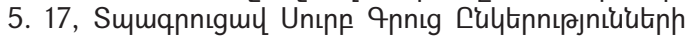
бutuunun, 1990p., 52 68-71:

2 4uin'umqhnp, F, 200-215, t, 3-18: 
huuunnpter, qhunulgylnou le upnter hul qnpouly nuर 5n, le hujunuh thu nnpteph

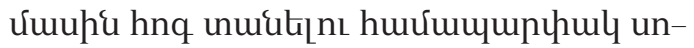

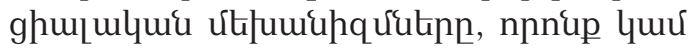

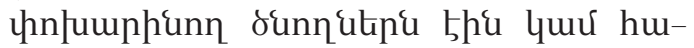

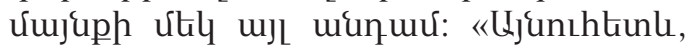
щuиนর์ unkưnıu utinulyuu puptiqnponsptum ujuuhup nựunàul uhuuntư: funt-

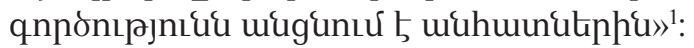

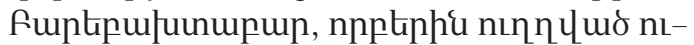

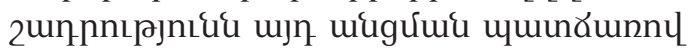

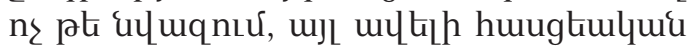

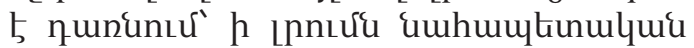
nuunuuhputiph úpqukpunujhu unpuoq-

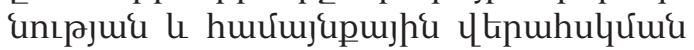
unghu[ulyuu uthumuhqưutph:

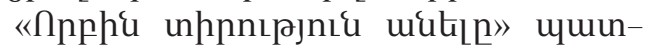

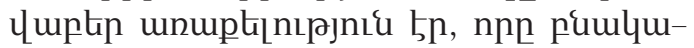

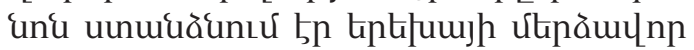
pupkluuर्u, hul npu uuhumphunıрjuи

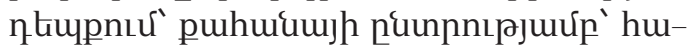

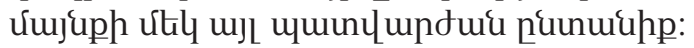

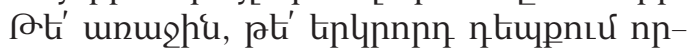

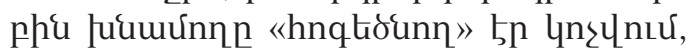

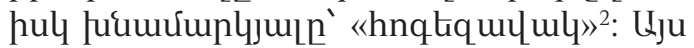

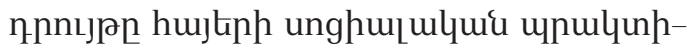
qujh uipulyuth ưuu th nuptiph nupugpnsu' nunhnsu uhusle Cujuuunuiap

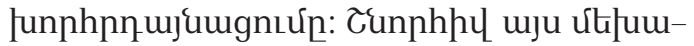
upqưutph' úfucl tintinup úto nnpuiung-

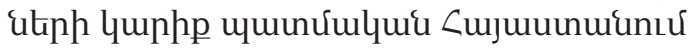

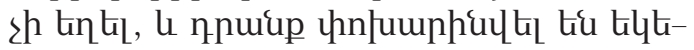
ntighutiphe lhg nunngutipnu, npuntn nsumutil tiu duul npptipn, lu npnug hn-

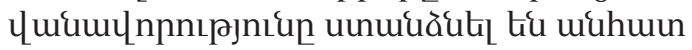

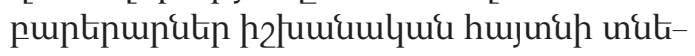
nhg:

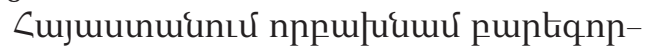

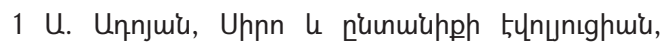
tplumi, <mjuurnuiu, 1990p., 52 5:

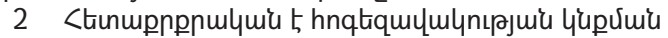

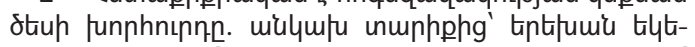

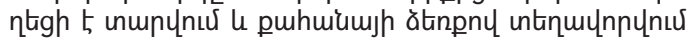

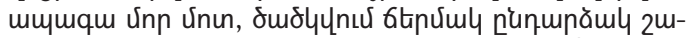

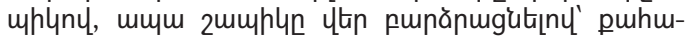

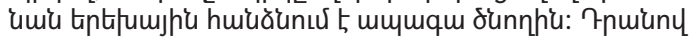
nnp'u mృluu nnf \&5, mృ hnqtiquuml:

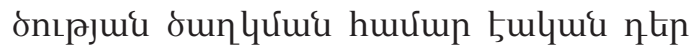
5 jumnugh (lu 2upntumlyntu 5 jumnul) ephumnutinıpjuid hnsulumo hpưumnup ulqpniuputiphg útyp. «Unıpnupt-

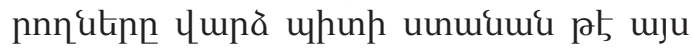

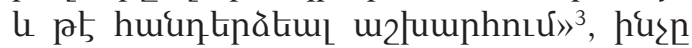

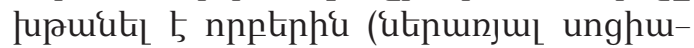

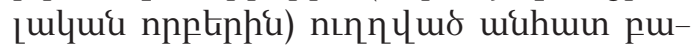

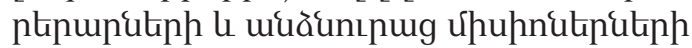

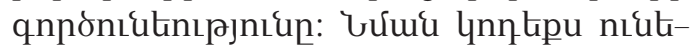

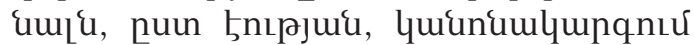

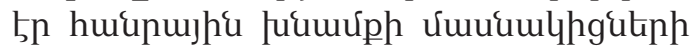
qnponsutinıpjnıun: উtputu Utô du-

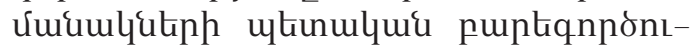

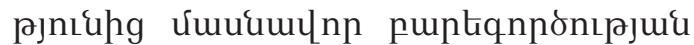
uugnıún pnıJl unltug npptiph unghu[u-

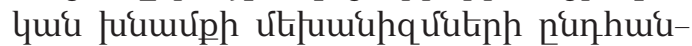

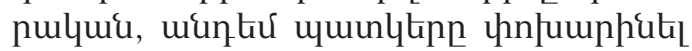

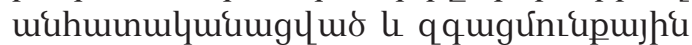
hnquunupnıpjứ̛́p": Lju uugnıún útp hpuluuuntpjuid utis hus-np unntúny

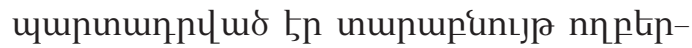

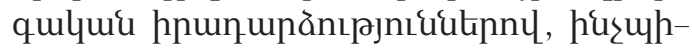

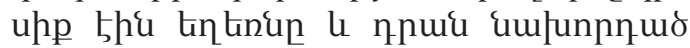

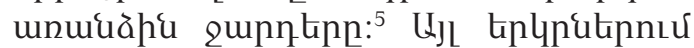

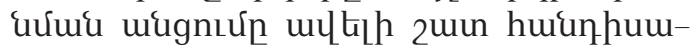

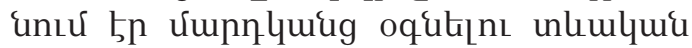

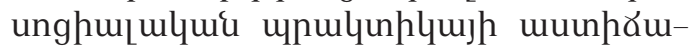

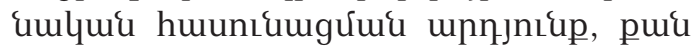
upunulump hpul haulutuphe htunlinn

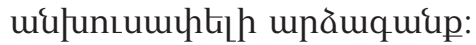

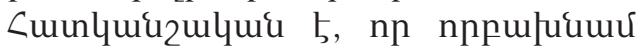
qnpontutnıpjnıup hujtph unghu[ulyuid

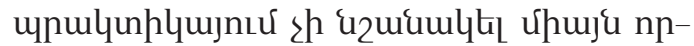

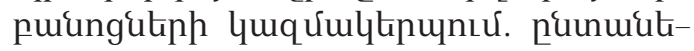

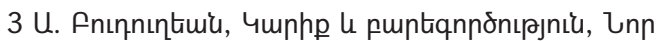

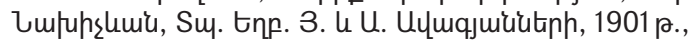
52 11:

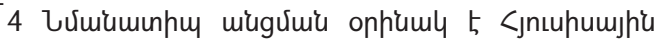

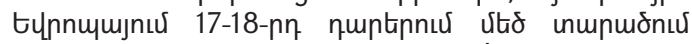

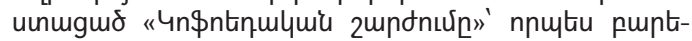

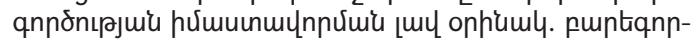

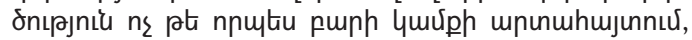

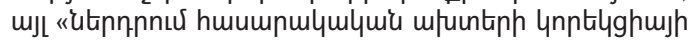

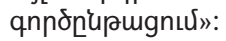

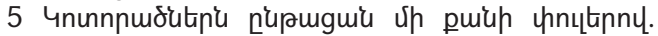

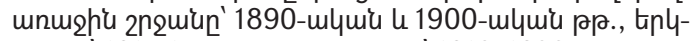

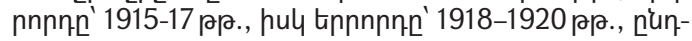

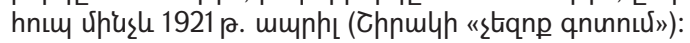




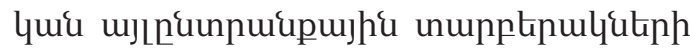

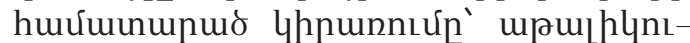

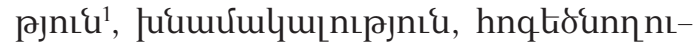

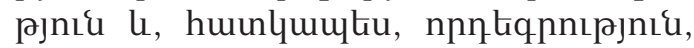

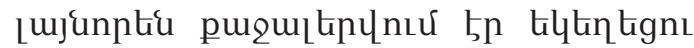

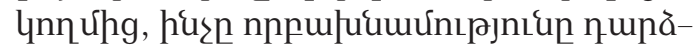

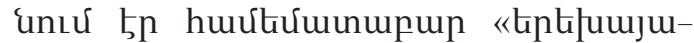

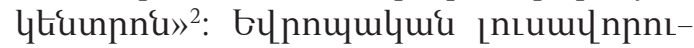
pjư htiu und

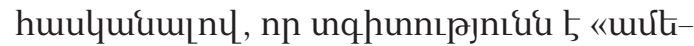

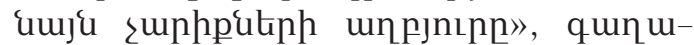

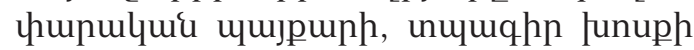
úhøngny huj hupntuunutiph puptiqnn-

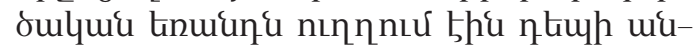

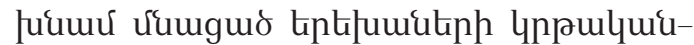

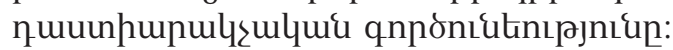

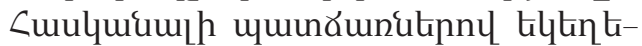

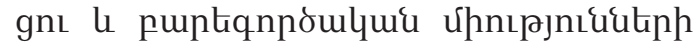

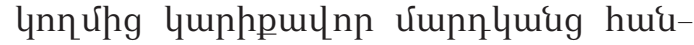

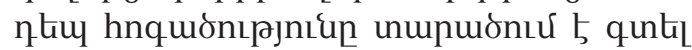

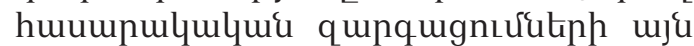

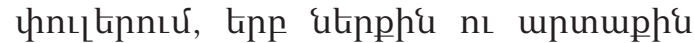
umuntipuqưutiph no ăqumduútinh ht-

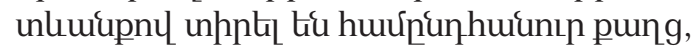

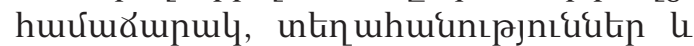

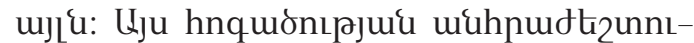

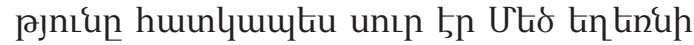

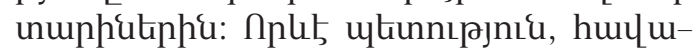

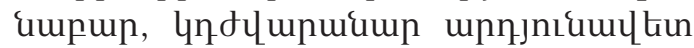
unàmquupta uц厶 nppuguro úuinılyetiph, puqưuhuqup

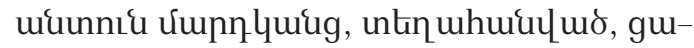

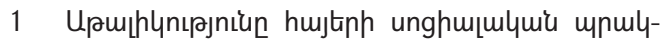

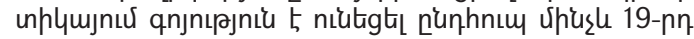
nuph ulhqph: Uju tipthujp hupuquin dunnh hu-

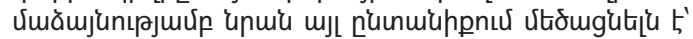

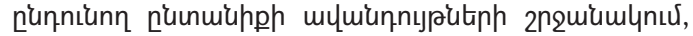

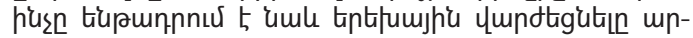

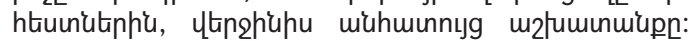

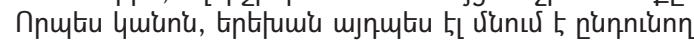

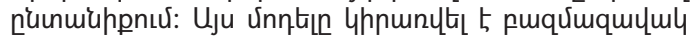

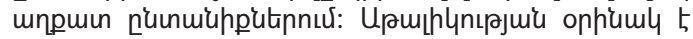
huiupuhujun 9ppnnh üuunúnıpjniun:

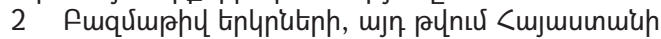

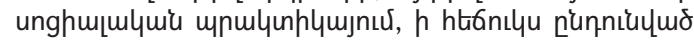
uhquqqujhe shumumprptiph qunumempmpununt-

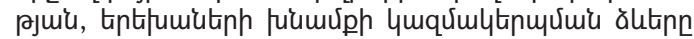

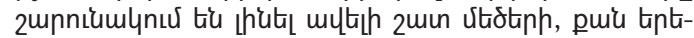

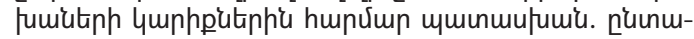

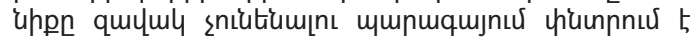
humulum [nı\&nıर:

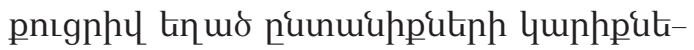

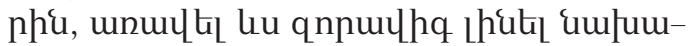

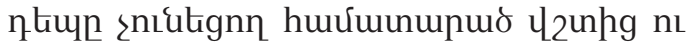
qnlumuputinh ununumnn unnıduóutinh

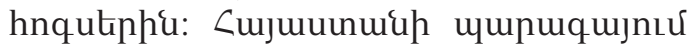

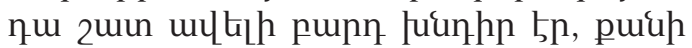
nn, ptul tiphpn hqne tiltintigh nifun, ujuntuưtumjuhu, snıutip utinulyuuntpjnıtu:

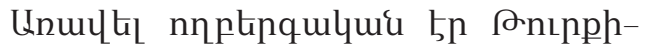

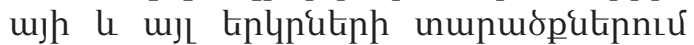
ưumguo npptiph aulquinuqhpn, npnug

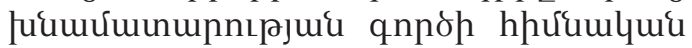
uunuuăunñ nunàmul mpnumuhưumuh

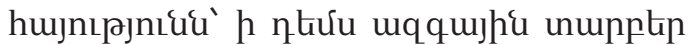

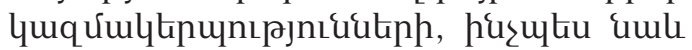
nnn2, huunnıl ujn Gumunulyny uintn $\delta-$

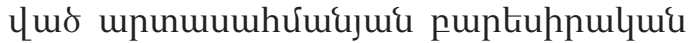

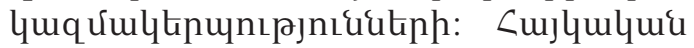

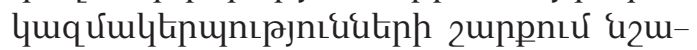

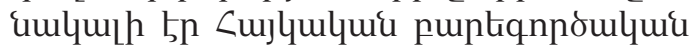

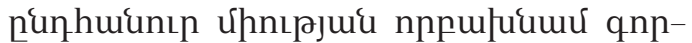

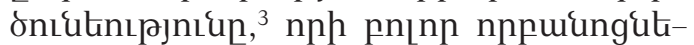
nnıu umutinn n乞 úpuju lnpnıpjnı́ tiu

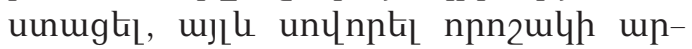
htuun:

npph unghu[ulqui htnuulquph huidptiu hpuluuu ưumuhnqnıpjnıú qnpơuu-

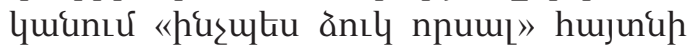

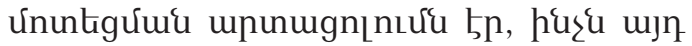
2n̨ưunıu ununtplnnnu pnınu qunqu-

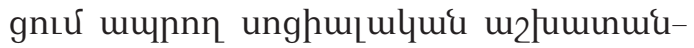
ph htúuujnıutinhg 5n: ઉnıju únuntgnıưu

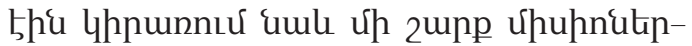

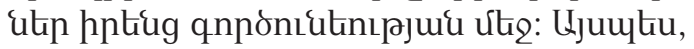

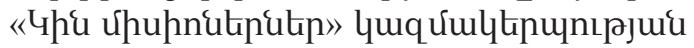
utplumumgnighs unpltiqugh tuunuph-

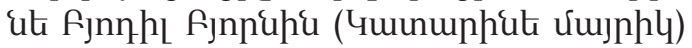

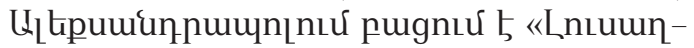

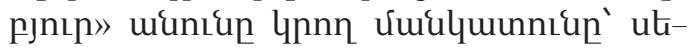
thulquu úhengutinnl hnq unuutingl 33 hus nppnılyutiph hưưn' unwghi uाuunsu nutinul npptiph qpunцuónıрjui

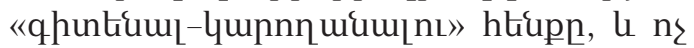

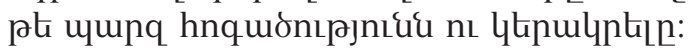

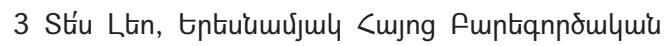

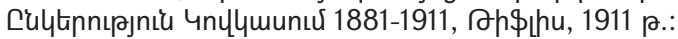


Uhus ujn, nupuulqphi, Unı2nıu wlyuduuntiup nundumpny lnunnpursutiph' Fjn-

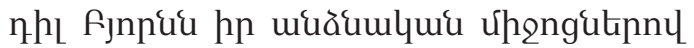

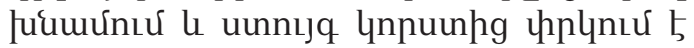
hupjnıpulnp tpkjumitiph ${ }^{1}$ : Lujunpté

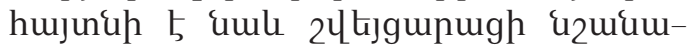

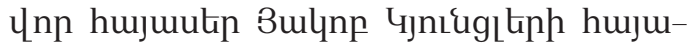

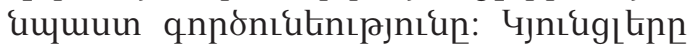

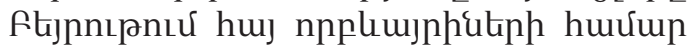
hnuuhunul 5 hpर्uantú, oduunulyntu úp

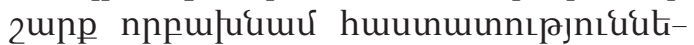

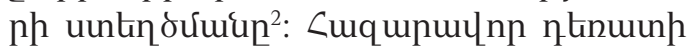

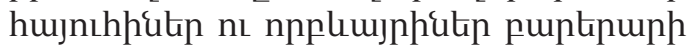

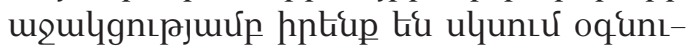

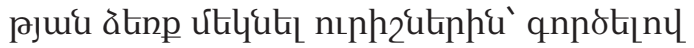

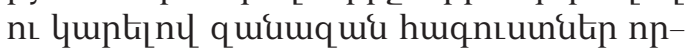
płph hưưup. qnpontutnıpjnı์ npu oq-

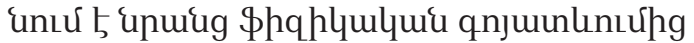

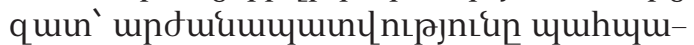

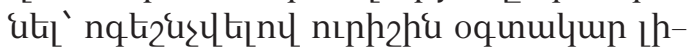
utipnt qqugntúpg:

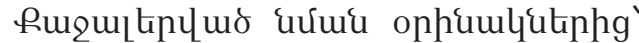

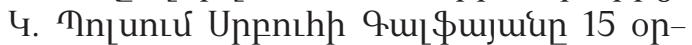
цu nupugpnsu tiltnkghuknh pultinhg, punuph unpuinhl pun wưuuknhg hu-

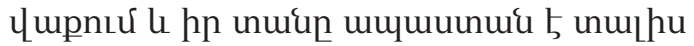
2-10 unuptyuu npp unghlutiph' unuiug lưuúpp hưump supuuting hp nne

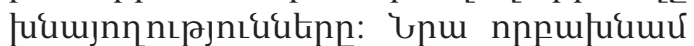

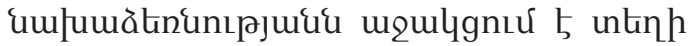

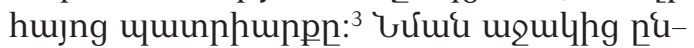

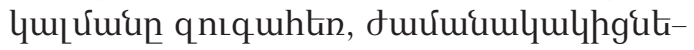
np unpupug nppuiungh úujpuwtiunhu hp2nıu tú qjnontuqunın, unukunniu nteqt-

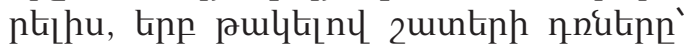

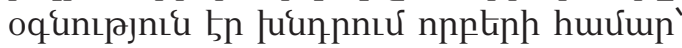
upduखumumpl amb ampumunhuph $\mathrm{nl}$

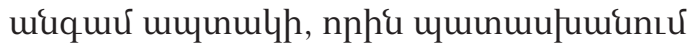

1 Stúu http://parsamean.blogspot.com/2011/08/blogpost_23.html

2 St́́u http://hayq.blogspot.com/2008/04/eventcommemorating-swiss-humanist.html

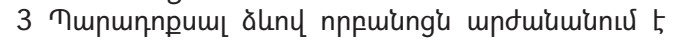

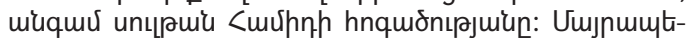

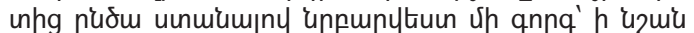

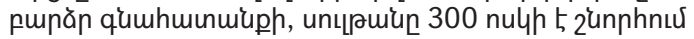
nnpuiunghu: tplymp unuphutatp unцpaiumlymu unuhg

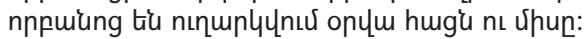

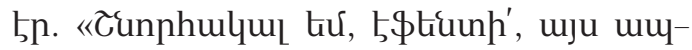
unuln hư pudhau 5 , humu butn a nnpt-

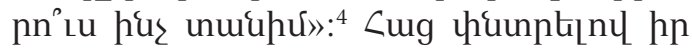
«jukn a nppknnı» hưưu' Uppnshhu, uj-

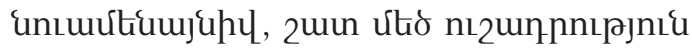
5 nupăunıư, np nppłpn unlnpkư yuu-

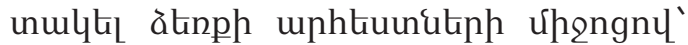

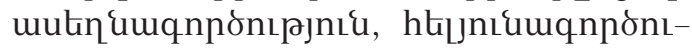

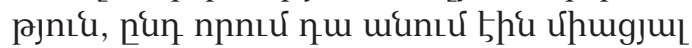

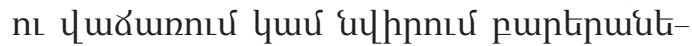

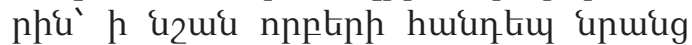
hnquónıpjuu hưưp tipuluunuqhunns-

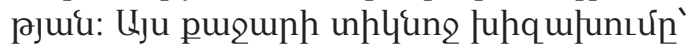
umzunumukint quphph utis hujunuluo

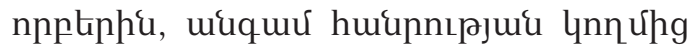

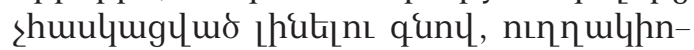

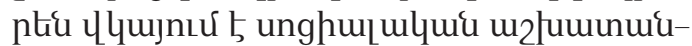

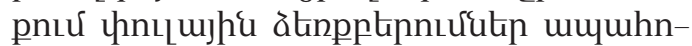

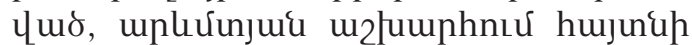

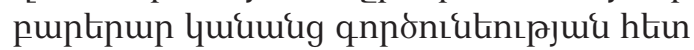
qniquhtinutiph úuuhu:

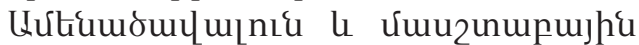

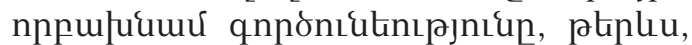

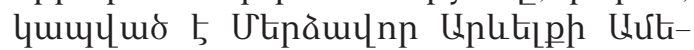

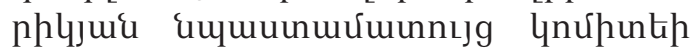

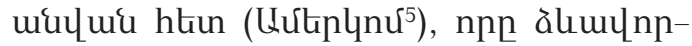

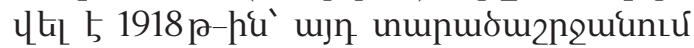

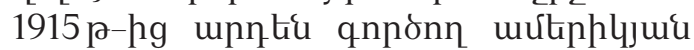

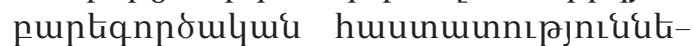

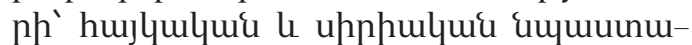

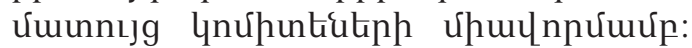
Lujng gtinuuumuntpjnisuhg hpuzpny

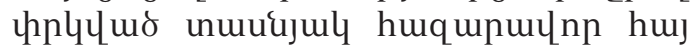

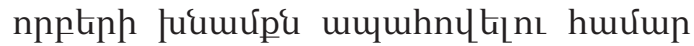

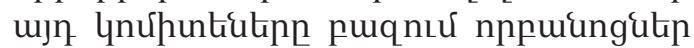
फhu umhnıu Uhphujnıu, Cntumumuunıu, bqhưnnunıर, Tum tuunhunıu, 4nu-

4 https://krishayas.wordpress.com/2010/04/15/

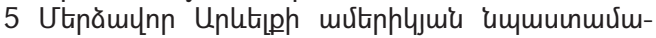

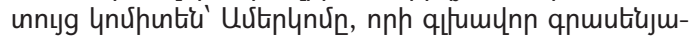

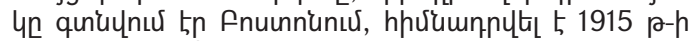

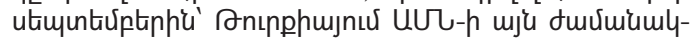

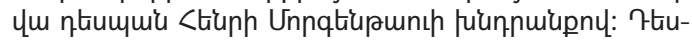

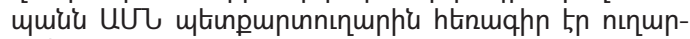

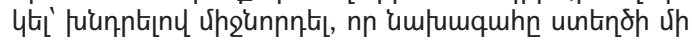

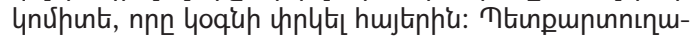

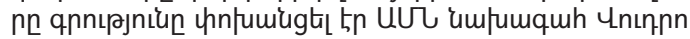

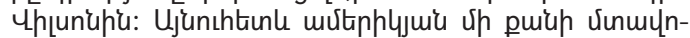

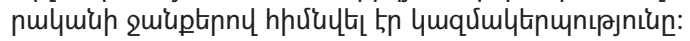




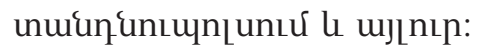

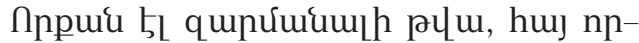

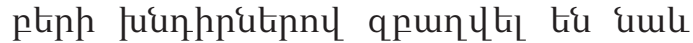
कnıpphujh qunulupnıpjntúu nı upu unưuăhu unuquanpnutunn: กpn2 unpjnıputip цlyujnıu tiu, np uנn «puph

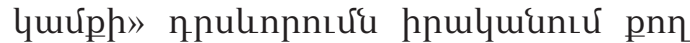
5n' pupgutinn npp ưuuguo tiptipumutnh quiuqumoushu úuhútnulyuumgntún («uupunul tntunu»): Ujnıu linnúpg, npp

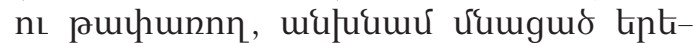
buuktiphu úty untnntu huluptin qqne-

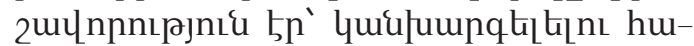

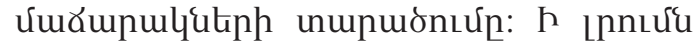

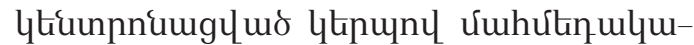

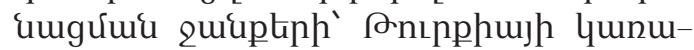

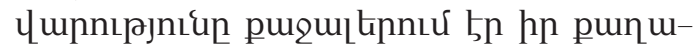
pughutiphu unue w2tuwinnnh, puinn

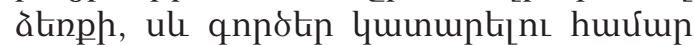
nunntukl huj npptphu, nuptip unlh ni huर्üawpulqukp umunawnny numuumunulus thu umhluid. qhup uwh-

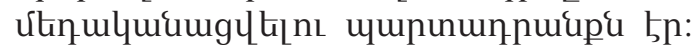
UJl lunupnl' 'juưph hnulntup' hupunt-

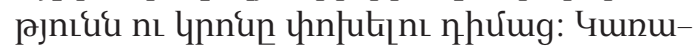

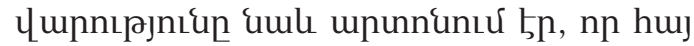

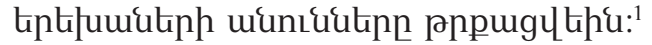

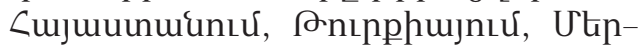

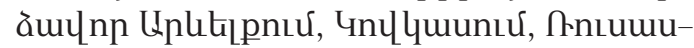

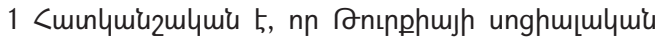

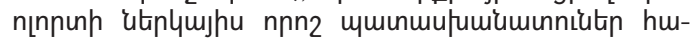

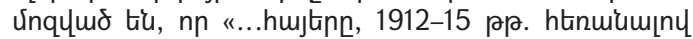

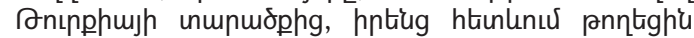

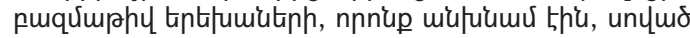

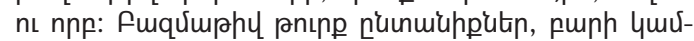

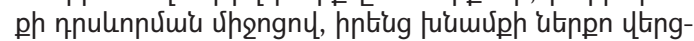

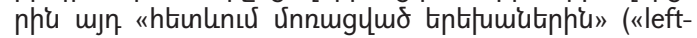

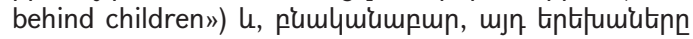

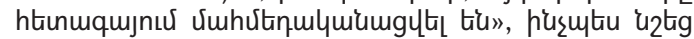

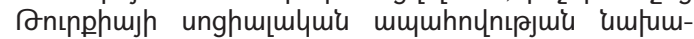

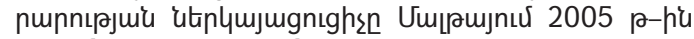

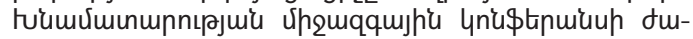

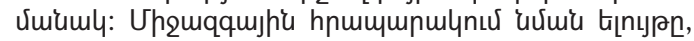

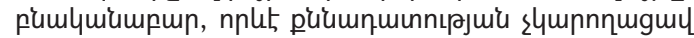

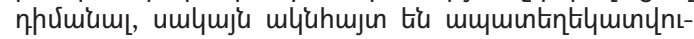

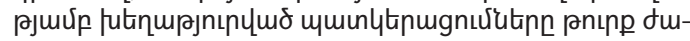

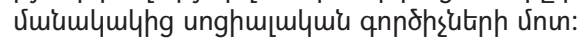

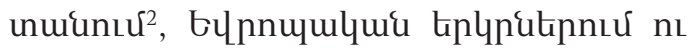

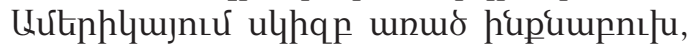
hulunınu nppuluduux le nppuhulup

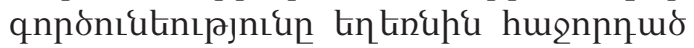

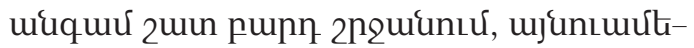

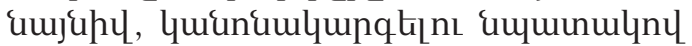

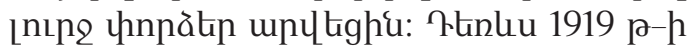

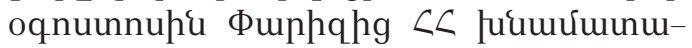

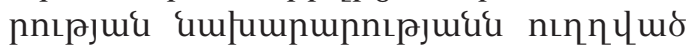

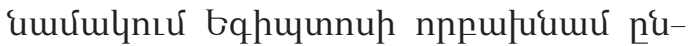

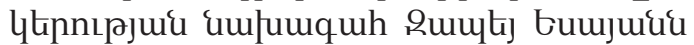
uju दupohpu niutip, np huj npp tipt-

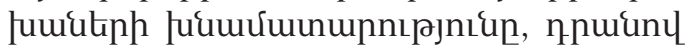

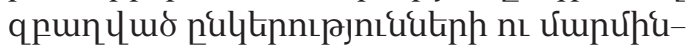

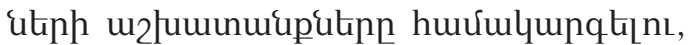

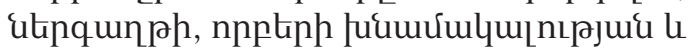

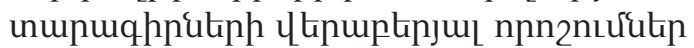
qujugitins qkpumuunh hpul nıupp

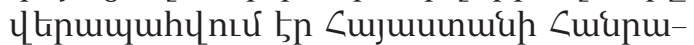

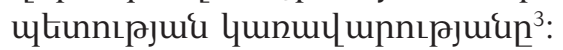

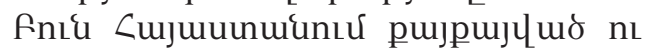

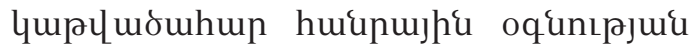

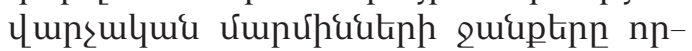

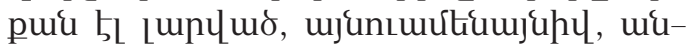

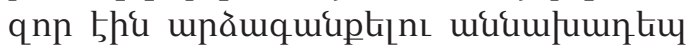

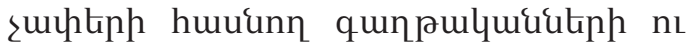

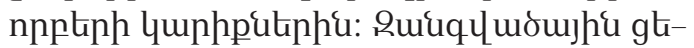

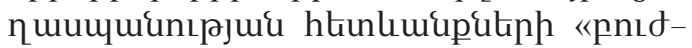

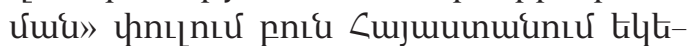
ntigns, ưnulnpulquinıpjuu, uqqh

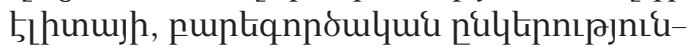
utph quplinpuqnıju úumhnqnıpjnıup

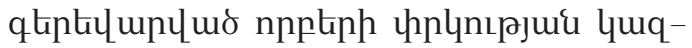

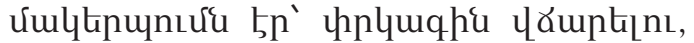

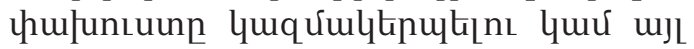
úp̨ngutipnu:

กppuiungutiph ưupupunup nup-

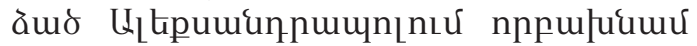

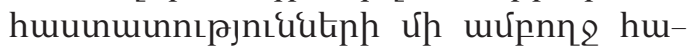

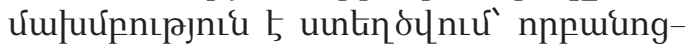
ukp, npuigg lhg úuulquumputiqukn, nunngutp, пunng-uphtuunuungutp,

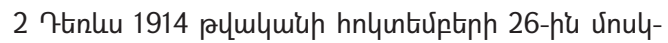

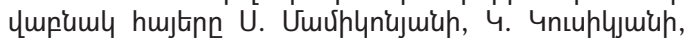

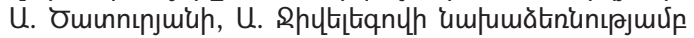

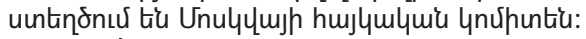

3 St́u http://serials.flib.sci.am/openreader/merc arev_6/book/index.html\#page/102/mode/2up 


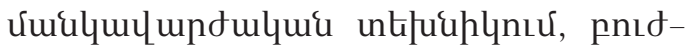

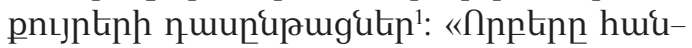
nulppulqui unuplumuknhg quin unцnnnıu thu quiumquid uphtiundin, ounup

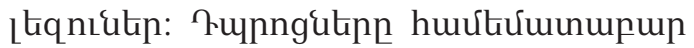

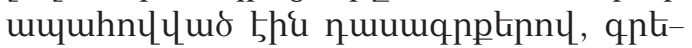

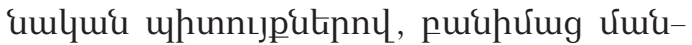
quulupdutinnl: Ljuuntin úto nı2unpns-

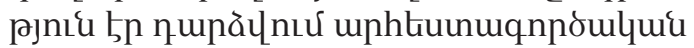
4nрnıрјuun: Ophuml' qunsưntigh ơu-

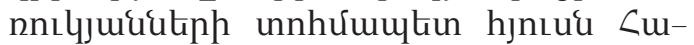

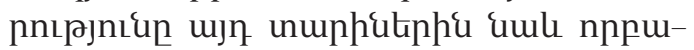
ungh uphteunuingh yuputiune $5 \mathrm{p} \mathrm{l}$ hp uphtiuun $5 p$ unlnptgunıu nnptphu:

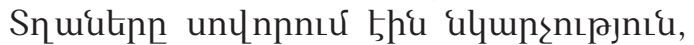

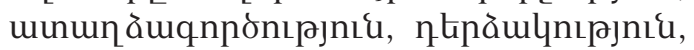

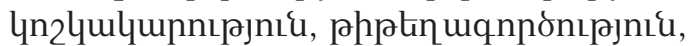

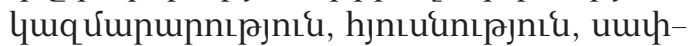

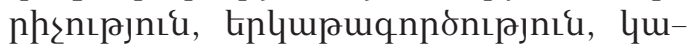

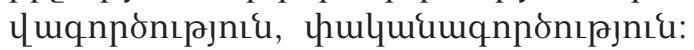
Tnıhqnup npptinp uthulqui nodtenny

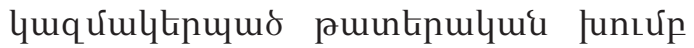
nutuhu: Unghl npptenp hưumuntu bhu

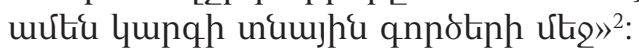

«Uútplnúp ưudquinutiph» umitnh htin w2lumunump hunul quintum-

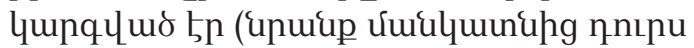

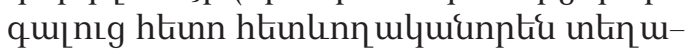

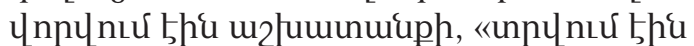
uphtuunh»): Bnıpophumb 5 tin ti Gule ujn

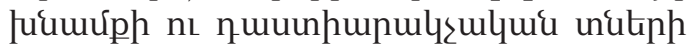

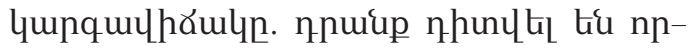

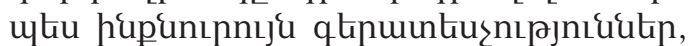
uquunltl tud huplikng, lupnn bhu qukil qnıjp, unupuop, qunnıgk hhर्u-

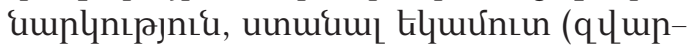
auuputiph quqưultpuntúng li punt-

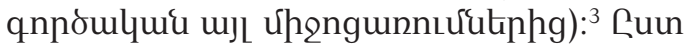

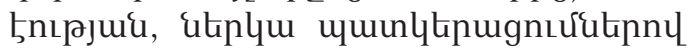

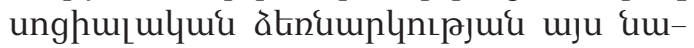

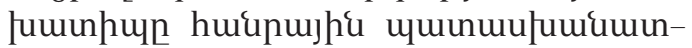

1 Stíu http://www.armin.am/images/menus/2033/ Hovsepyan_E.pdf

2 http://hetq.am/arm/news/44812/aleqsandrapoliorbanocnery.html

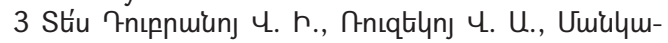

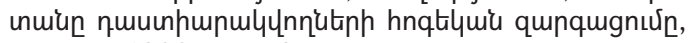
Unulum, 1988 p., 52 9:

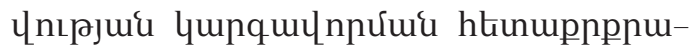

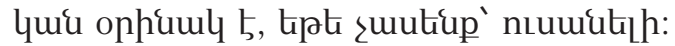

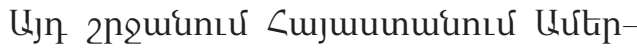

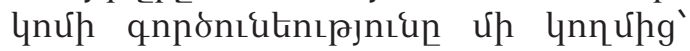

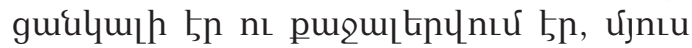

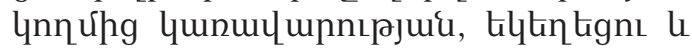

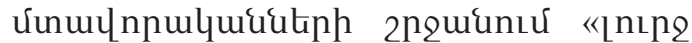

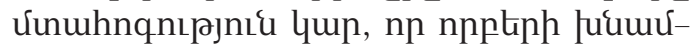
phu qniquhtin' ounup uhtephg dưư-

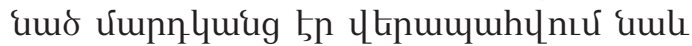

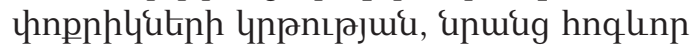

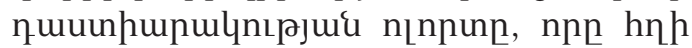

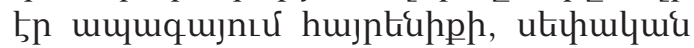

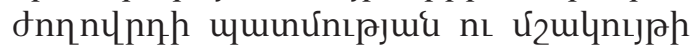

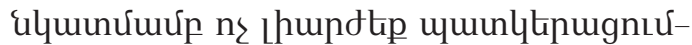
utp noukgnn punupughutp lnptins l

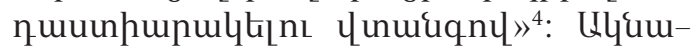

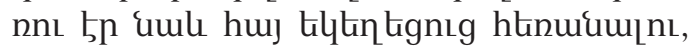
pnnnpulyuu hujugputinnl unnqnpltını

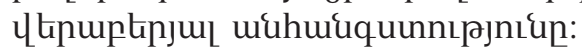

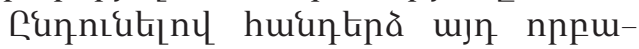
ungutipnıu दhpununn únuntegnıưutiph'

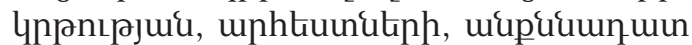
únunkgúuu, unnne punnjuhnqtpuiuu-

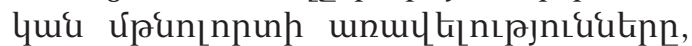
npnup ppumitu umuunntu bha nppt-

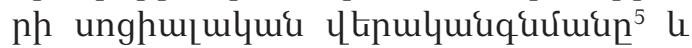
umunpuuunntú upuiug htinuqu hupuns-

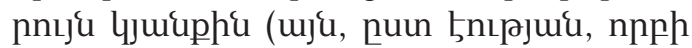

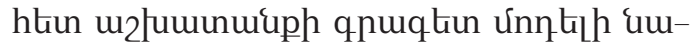

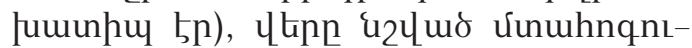

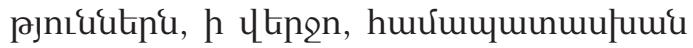

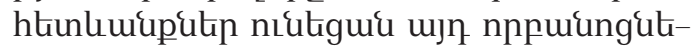
nh huúum:

Iunphpnujhe h2luwuntpjuid huunuunnıúpg htinn pupkqnpoulumu luquu-

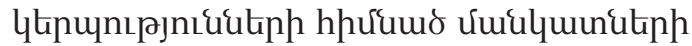

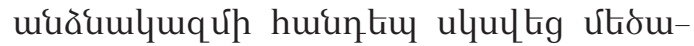

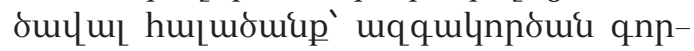

\footnotetext{
4 http://www.armin.am/images/menus/2033/ Hovsepyan_E.pdf

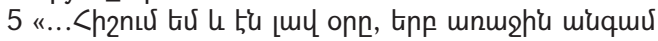

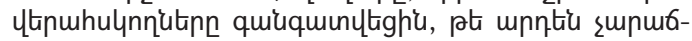

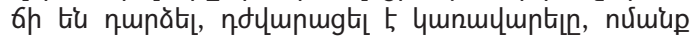

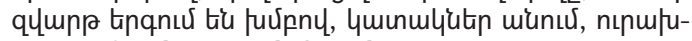

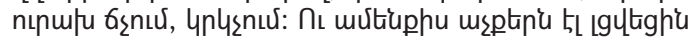

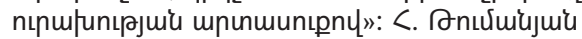




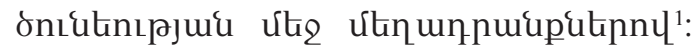

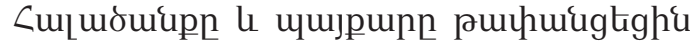

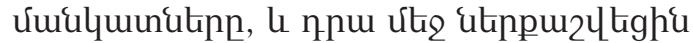

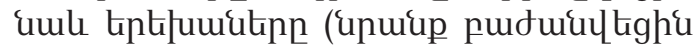

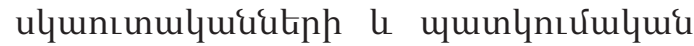
enluunutiph):

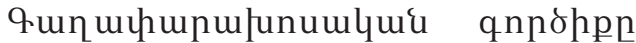
uluktg qnpotil n々 úpuju nppuiungutipnıú.

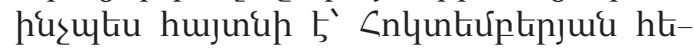

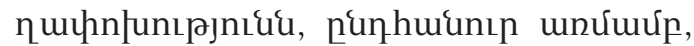

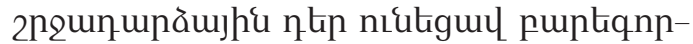

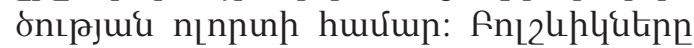

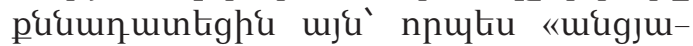

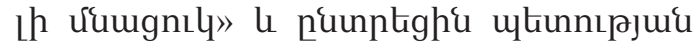

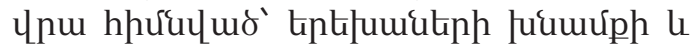
unghu[ulyur oquntpjux «lquinupk]u-

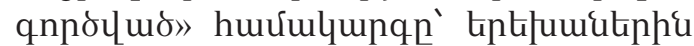

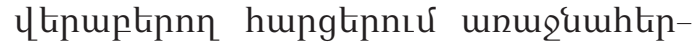

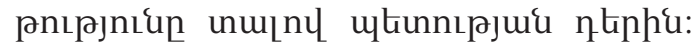

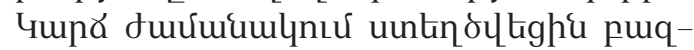

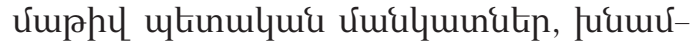

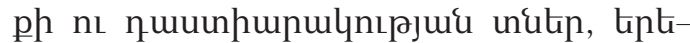

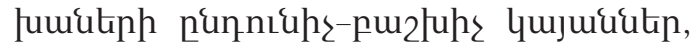

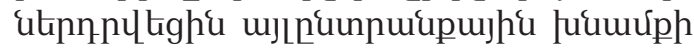
htinuppphp unupptinulutip:

npp le unghu[ulquiu npp tiptuwutiph

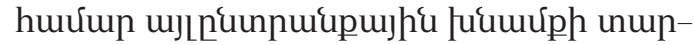

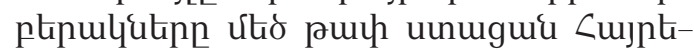

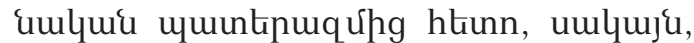

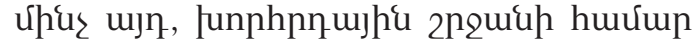

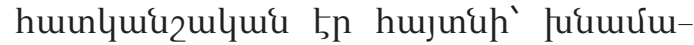

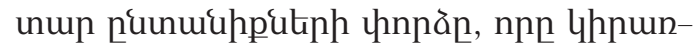

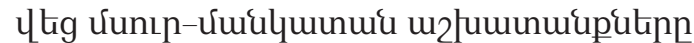

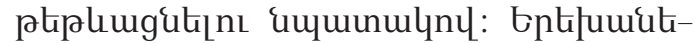
nh luphputiph hưưp úpønguten hulupuqntins घumunulynl umpptinu-

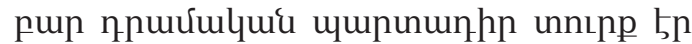

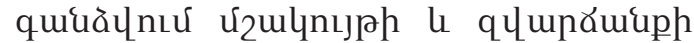
oquituknph: Uju घumunulyny uiqquर्u

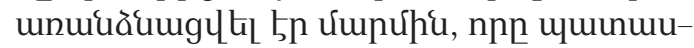
Jumumunns 5p ujn unnıpp hulupuqnumu hưum: Luoplewe tpktumutiph bưnhnuknnl qpuny umgltighu hứumumumulumu qunptip'

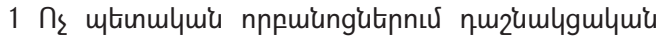

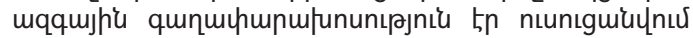

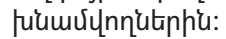

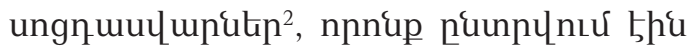

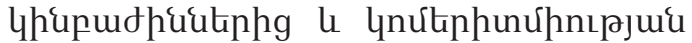

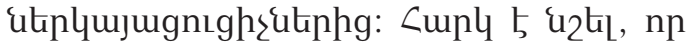

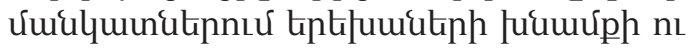

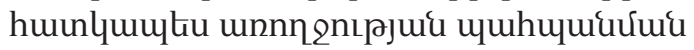
jưnhputinn hinns thu nunntutilh uu-

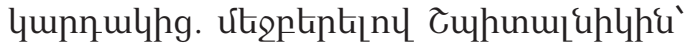

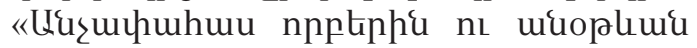

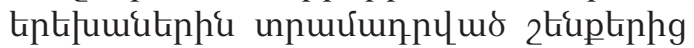

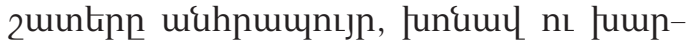

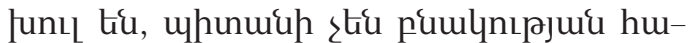

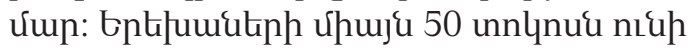

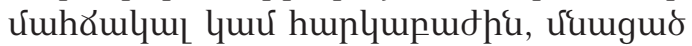
50 unnlynup umunquuku punıu 5 humu-

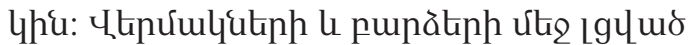
5 lunumul lunun: $\bigcap_{2}$ pninp tpti|umukpu nı-

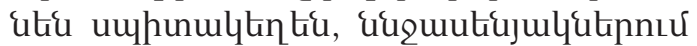

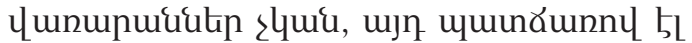

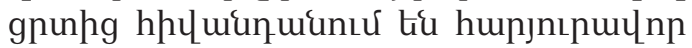

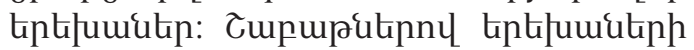

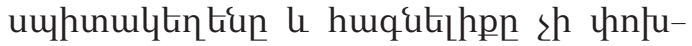

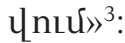

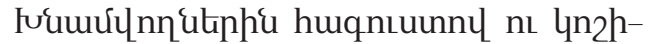

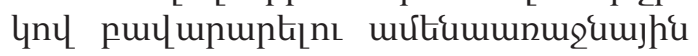

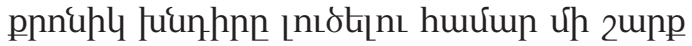

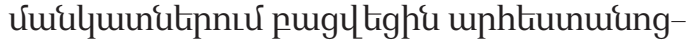

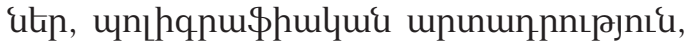

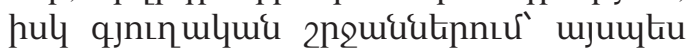

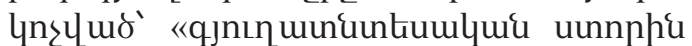
unhuh nunngutp»»' ujnuhunl wuwhn-

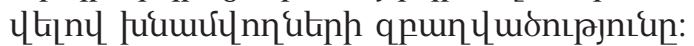

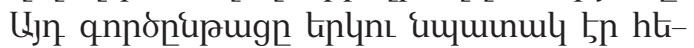

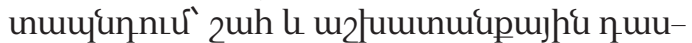
unpupulntpjntu: Cujntumuludu umuntpuqứhg hłinn $\angle U U<-$-nıu qnjnıpjnıu niutep

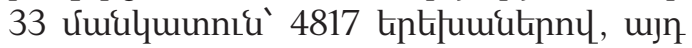

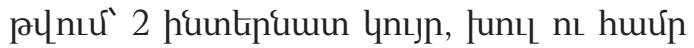

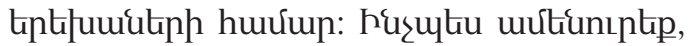

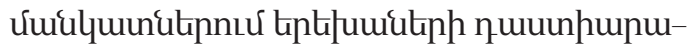

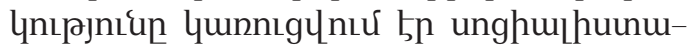
quiu huumpulyunqh qunuhupulununs-

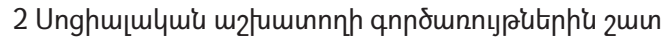

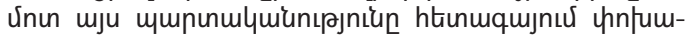

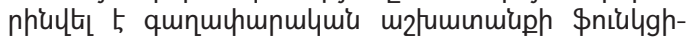
munu:

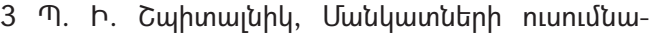

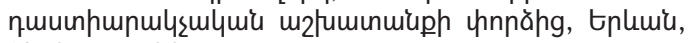
1953 p., 52 38: 


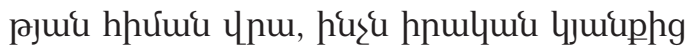

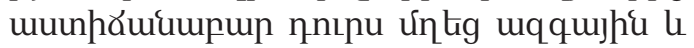
hnqunp nuuunhupulnıpjnıun:

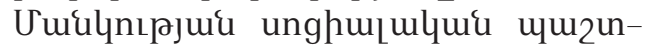

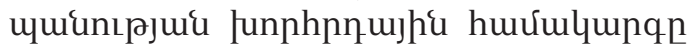

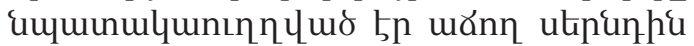

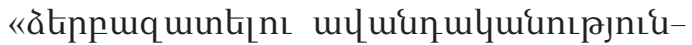

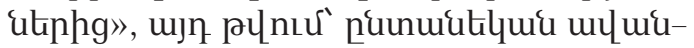

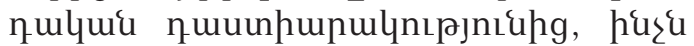
hpulquiugitins hứup qunuthu-

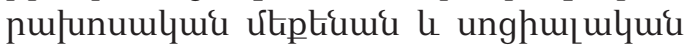

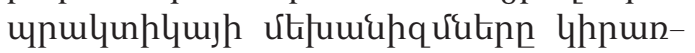

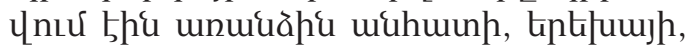
hưumuph le wyth umu2unupushu hup-

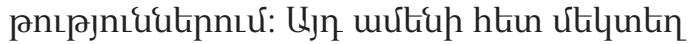
umb uy uquqnujup huugltighu tiptjum-

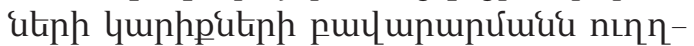

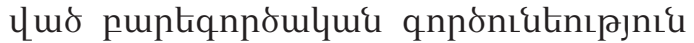
ঠшцщц

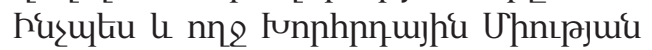
unupuopnıu, 60-70-uluuuutiphu Cujuuunuunıu, uté purh unuguru humnnly qh-

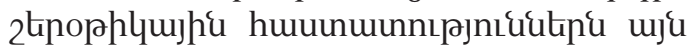

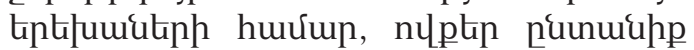

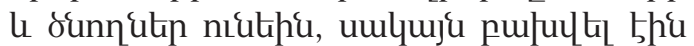

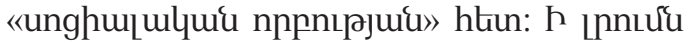

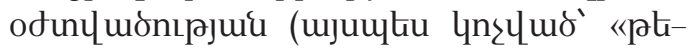

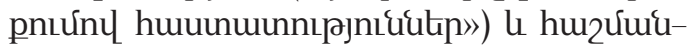

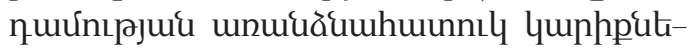

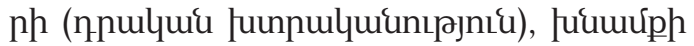

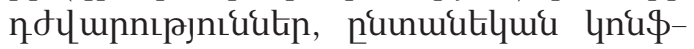

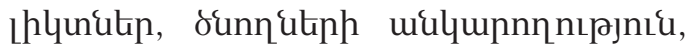

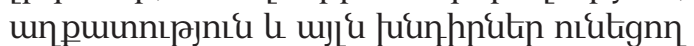

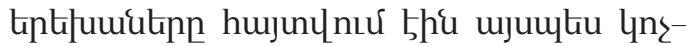

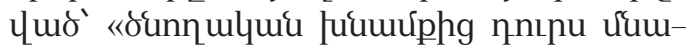

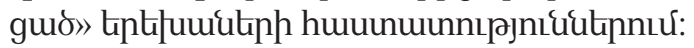

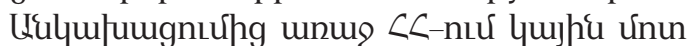
65 qh2knophluushu huuumunnıpjnıudun:

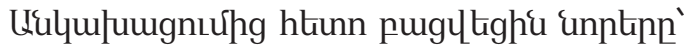

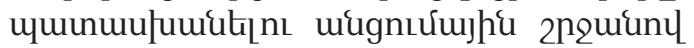

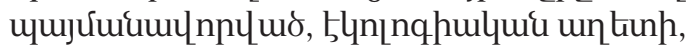

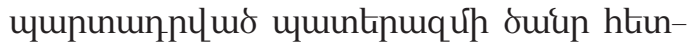

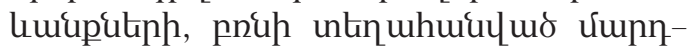

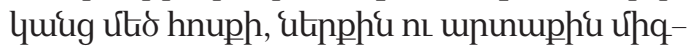

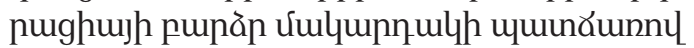

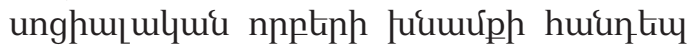
htinq htint wănn umbuuquplyhu:
Chunpunphpnujhu 2nøuuh ununtuuulyuu le unghu[ulyui huर्umlumptph aqumdu-

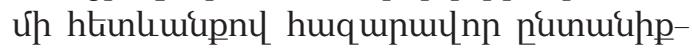

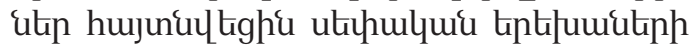

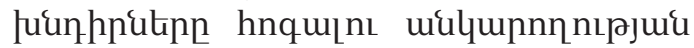
huunhưud: 'supulhu hưumlyunqhg htunn nthluu umhumulnn qh2knophlyujhe

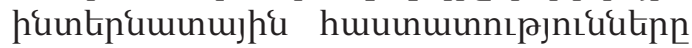

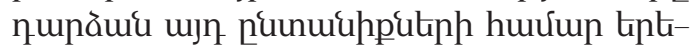

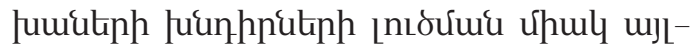
nuinpuipe (1988p-hu qh2tpophluustu

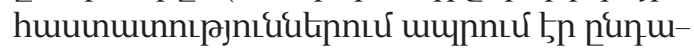
útun 1000, hul 2002 p-hu' 10.000 tiptuu,

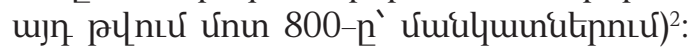
bpt umpulhunıu ujn huuunuunnıpjnıu-

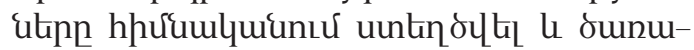

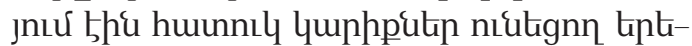

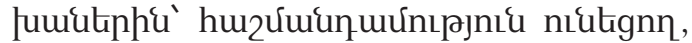
unupptip odunluónıpjntưtep ntukgnn,

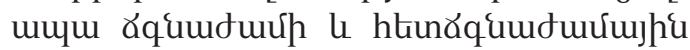

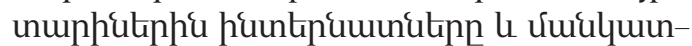
utpp ulukghe mumuunuati unpuin nt

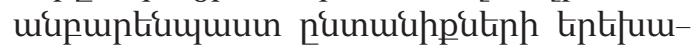
uthpu' unghuцuluiu npptphu:

1988 p-h tplnw2undhg htinn puqúmphy úhॄuqqujha le uhjnınph pupt-

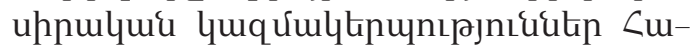
juuunuid ptiptighu ns úpuje ujnıpulyui oqunıpjniq, ujlle unghujuluu, hnqt-

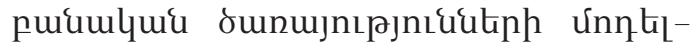

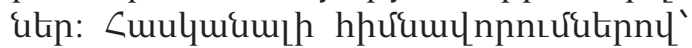

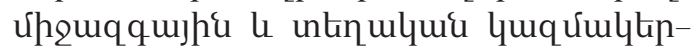

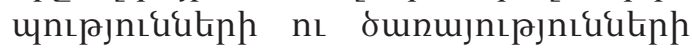

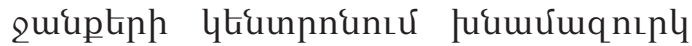

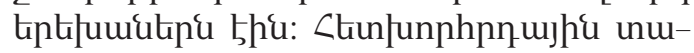

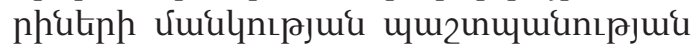

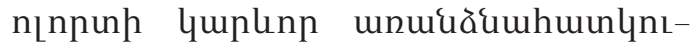

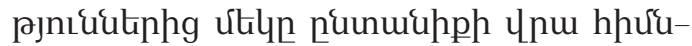

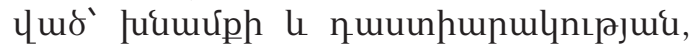

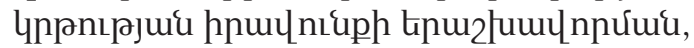

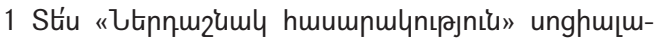

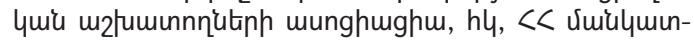

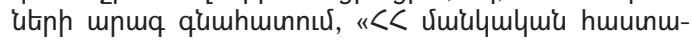

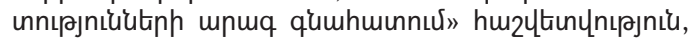

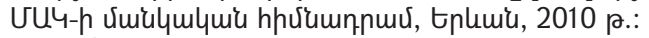

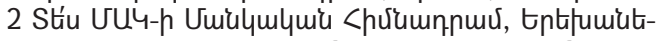

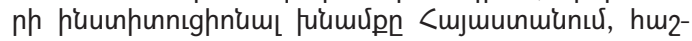

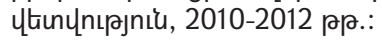




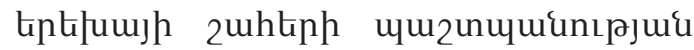
puqưupuntjp, n々 ukinulumu omnujnt-

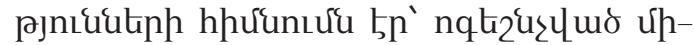

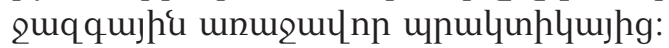

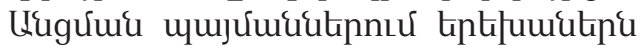

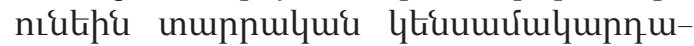

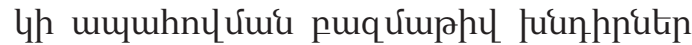

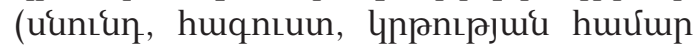
uuhpudtizu umumquitin, pumlqunu-

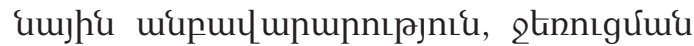

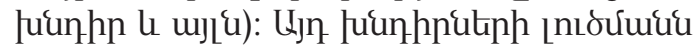

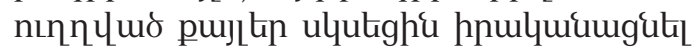

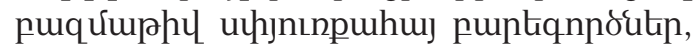
uhjnınpuhtu purkuhpulquiu luqqưulkn-

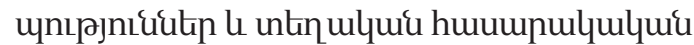

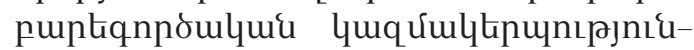

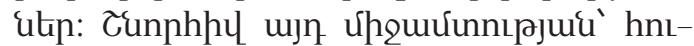

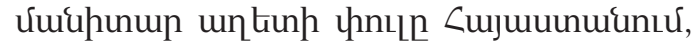
qunotiu ph, hunpuhupltig, umluusu unp

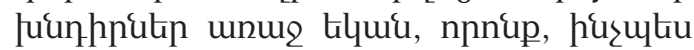

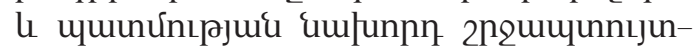

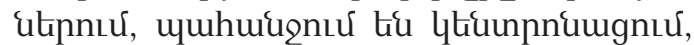

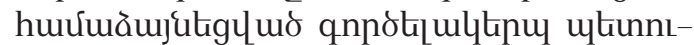

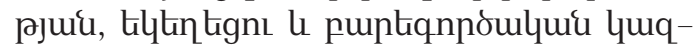

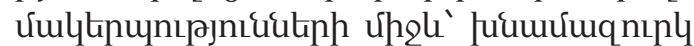
thitumutinh buunhnutinhu qnuqtin up-

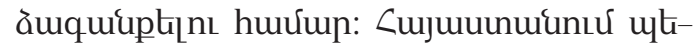

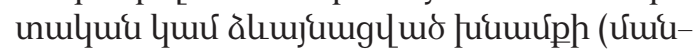

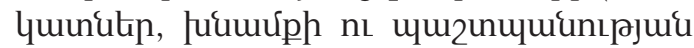
huuunuunnıpjnıরukn) ukppn quiulnn tiptipumutinh úpuju útl unnlnue 5 nnp': rpuid hulqunuly, teplnntu unuptgunuph wơnıu 5 «unghu|ulquu npp» yuư nuunu-

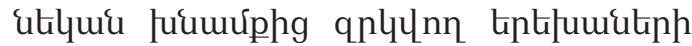

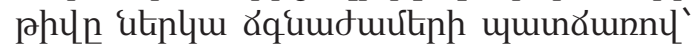

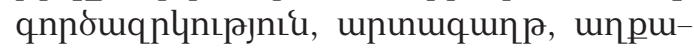

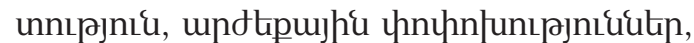

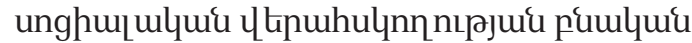

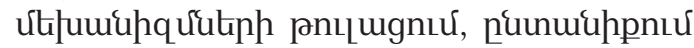
úpqutinunujhu hupuptennıpjnıuutinh unjumlthnunıú le m] u:

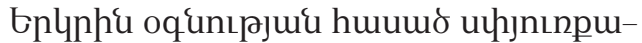

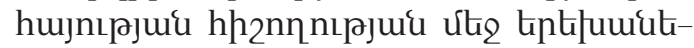
nh uju lhämln uunguguntu 5 tintinup

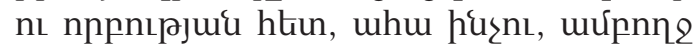

1 Stíu ưnı̌u untฤnnư:

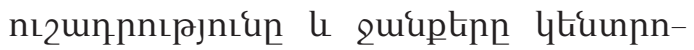

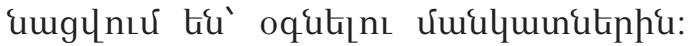

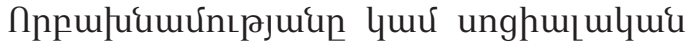

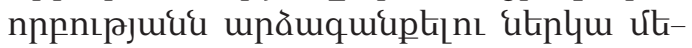
jumupqúutinn típulqu tí enjumlinu-

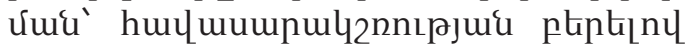

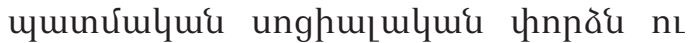

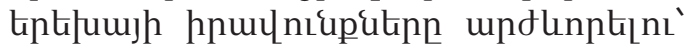
dưumumlymlhg unwøulnn unghu[u-

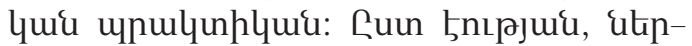

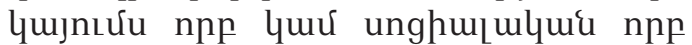

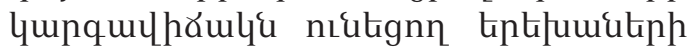
hưumen unlum tí juumúpp quqúultinu-

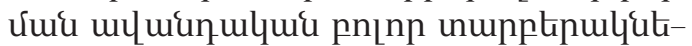

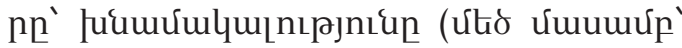

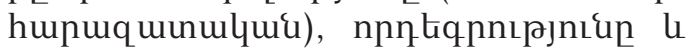

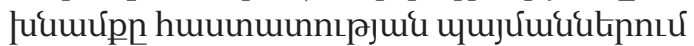

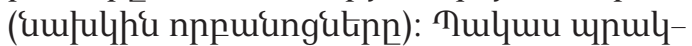

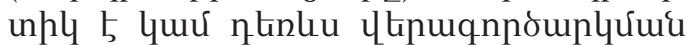

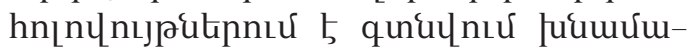

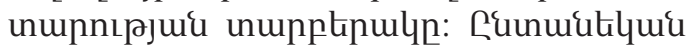

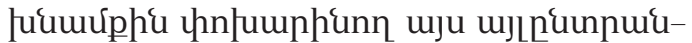

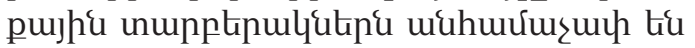

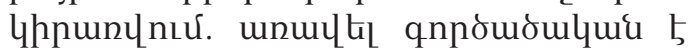
huunuunnıpjniuutiph unupptpulp, hu-

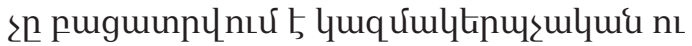

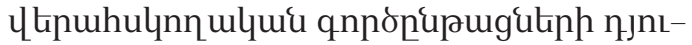

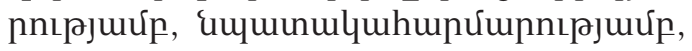

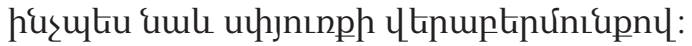
पthnghuu uuăunınug, uưưuugnnn, pujg

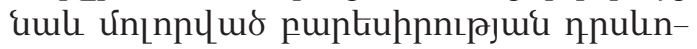

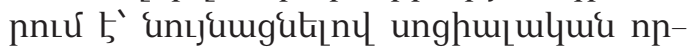
pnเpjnıun hpulqui nnpnเpjui htin:

Uuiqumitiph unupptipulntu qni-

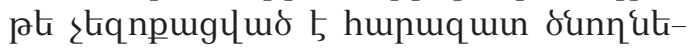

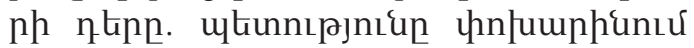

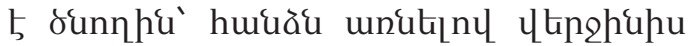
ưunumuluunıpjnıưtenn, úhųnth tipt-

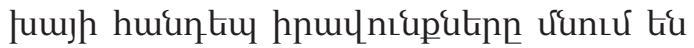

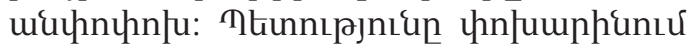

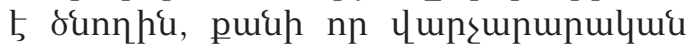
útipnnfuknny uuhump 5 oqukil, np ytun-

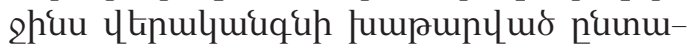

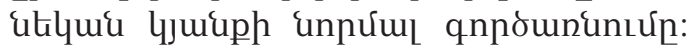

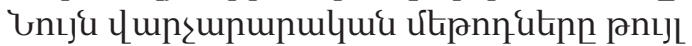

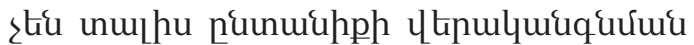

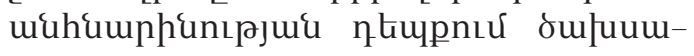




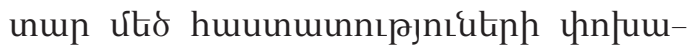

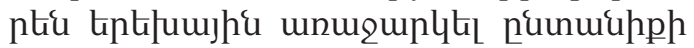

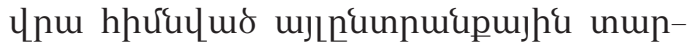

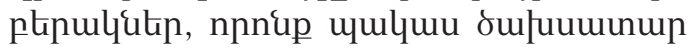

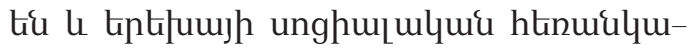

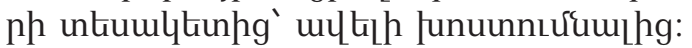

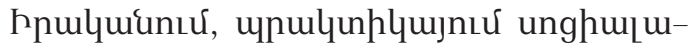

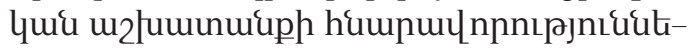

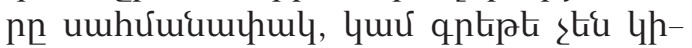

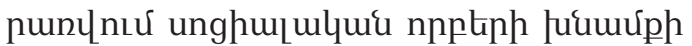

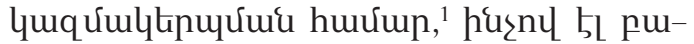
guinplnuर 5 numuappuhter luumúph

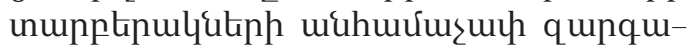

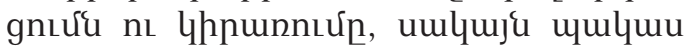

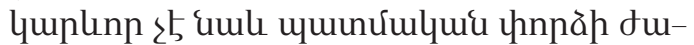

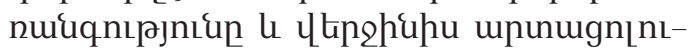
un utplyu qnpotiulutpuntư:

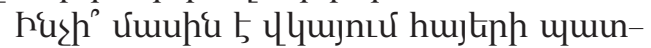
umlux unghupulum unulunhlyux:

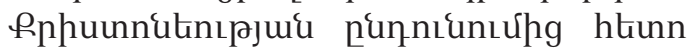

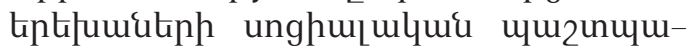

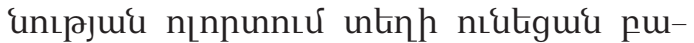

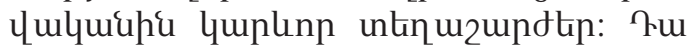
ptilnsưumshe 2nowi th tiptipume hpu-

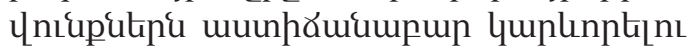

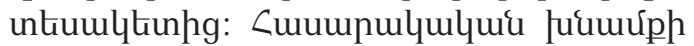

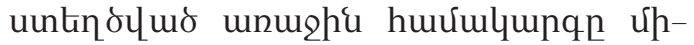

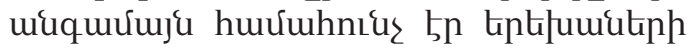

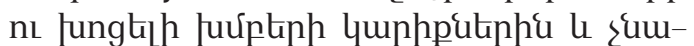

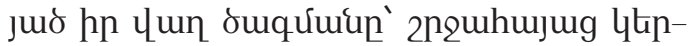

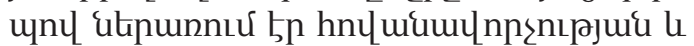

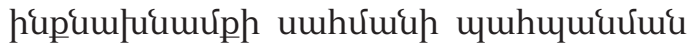

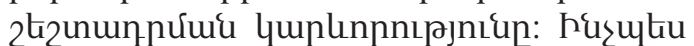

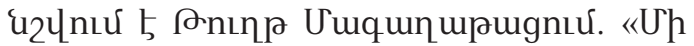
lknulpte, npnuhtinl punguo 5 , цtnulph, npulkuqh htinujuns punguo ¿lhup.... ${ }^{2}$ :

Cuulumumpl, np «Utiuml uptpulqui oqunıphıun, np unplnıu ๖ unuiug pună-

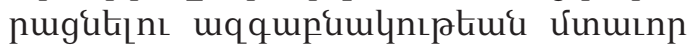

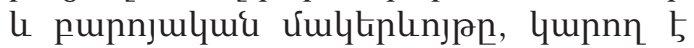

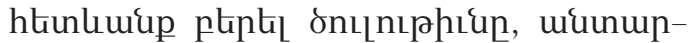

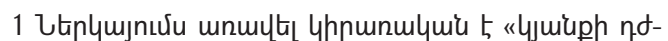

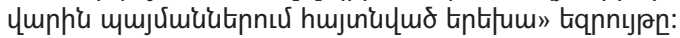

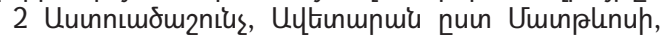
5. 17, Suquqpnıguy Unınp 9pnıg Culytpnıpjniütinp రufuunun, 1990 р.: ptipnıphıun, únıpugluúnıphıun, úp houpny pupptiph quqưųntơưun»",

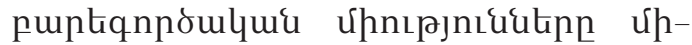
engukpu nınnnıu tha huunumuku npp

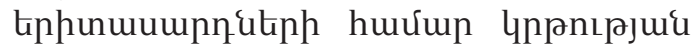

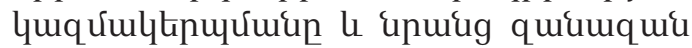
uphtiuunutiph nıunıgúuin, umluju uш-

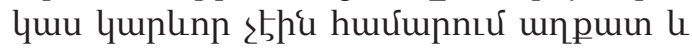

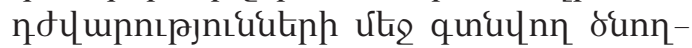

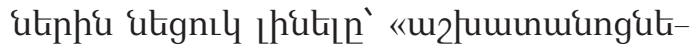

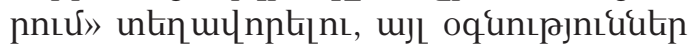

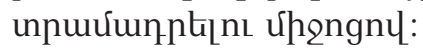

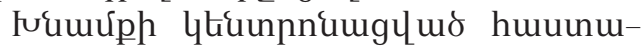

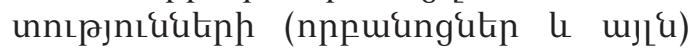

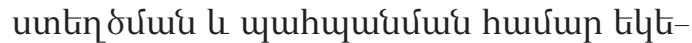

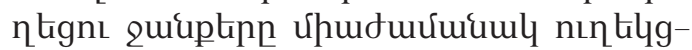

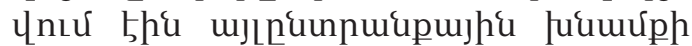
unupptpulukph pupnjulqui tupurưuru úhongutinny: bltintighu hnq 5p unuunıu, nputuah unưăhu hupumlyuu bưuúnnutph l. puptipunutiph huumpulyu-

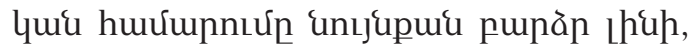
nppuiu nppuiunguteph hnц uuulunnutph-

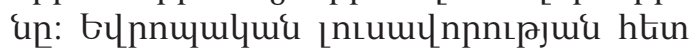

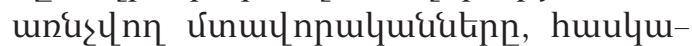

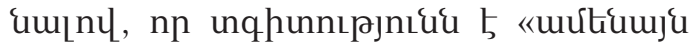

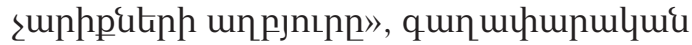
umupuph, unumuqhp lunuph úhengny hus

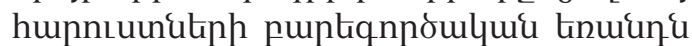
nınnnux thu ntiuh npptiph lnpulyuunuuunpunuls nph uumunuly úmplyuigg hupumpul qnpotint humpuulnpnıpjnıরutiph nuăt-

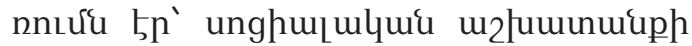
utplyu htumujniun ${ }^{3}$.

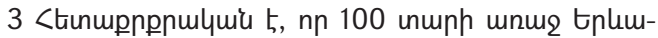

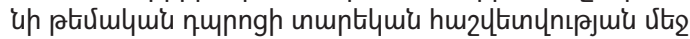

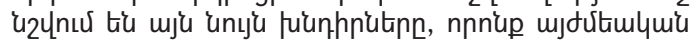

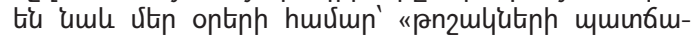

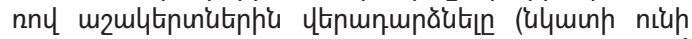

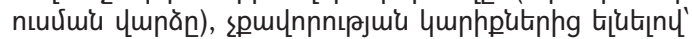

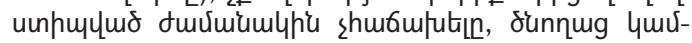

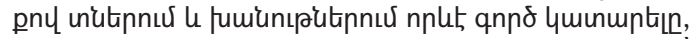

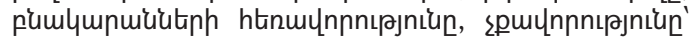

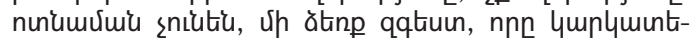

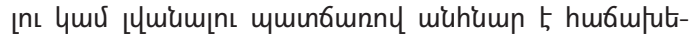

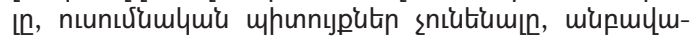

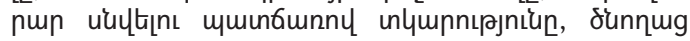

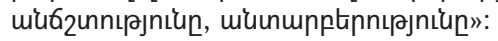




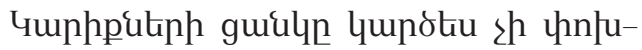

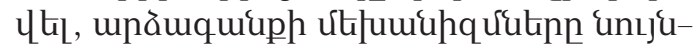

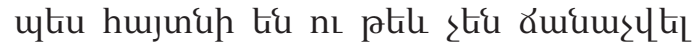
npuku unghwimumu w2 lumunumph

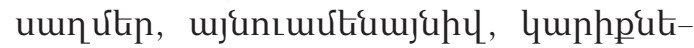

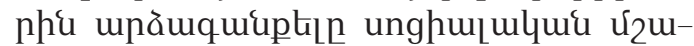

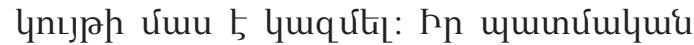

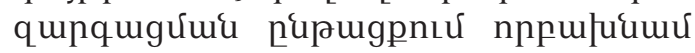

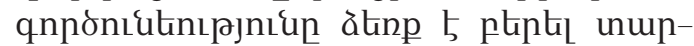

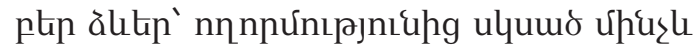

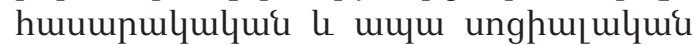

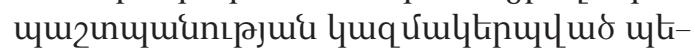

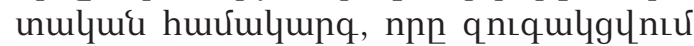
5 र́uumuln $\mathrm{l}$ huumpulqulum punt-

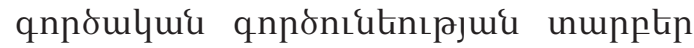
untumlyutiph hưưnpưue htin:

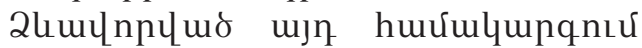

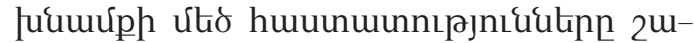

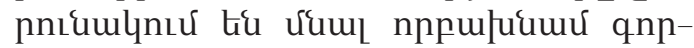

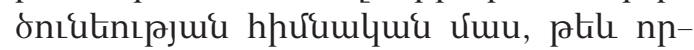

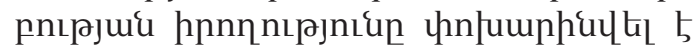

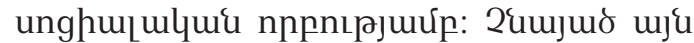
hưqqưưuphu, np nppuiung tiqnnıрр

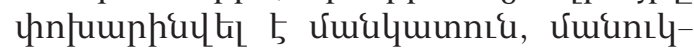

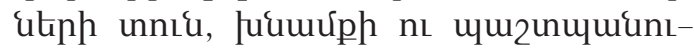

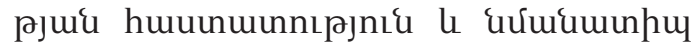

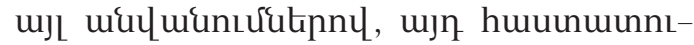
pjnıkutinh huumpulqulue nulum ntún zunnı́umlnnu 5 ưum nputiu nnpu-

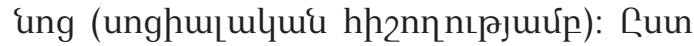

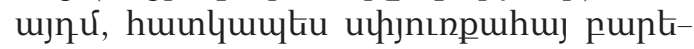

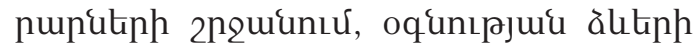

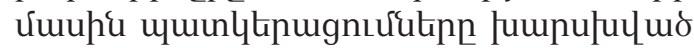

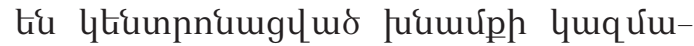

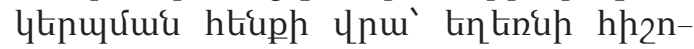

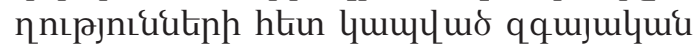

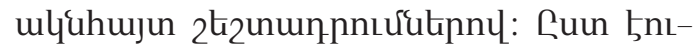

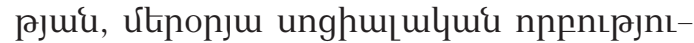

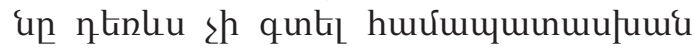
núlųnıư lu unàmquiup:

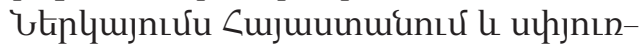

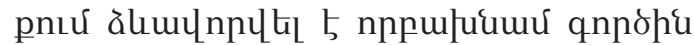

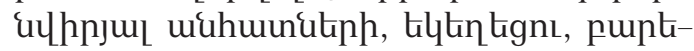

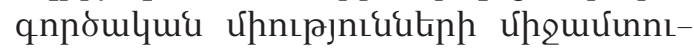

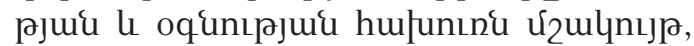
nnp huumpulqulqui aumurnıú 5 ptinnıu

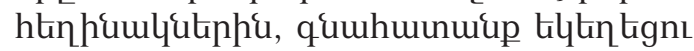

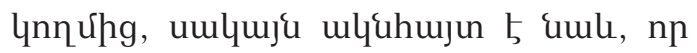

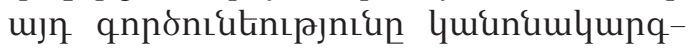

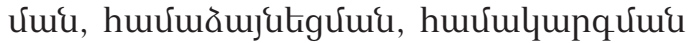
unıjupuí lumpp nıuh, nnpui nuntulqphu' tin tinuhg htinn nulquơ 2nquiunıú:

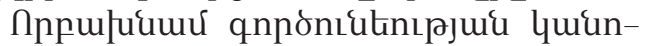

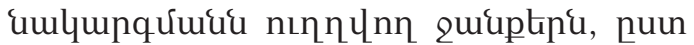

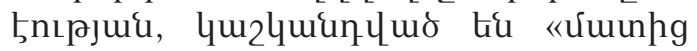
цnı2 huunn» pupkqnnowlyuu quqưu-

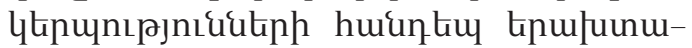
qtiu ytinuptinúntuph le npueuhg plunn

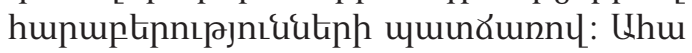

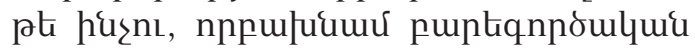

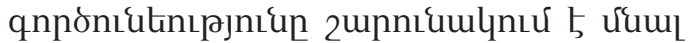
hupumpnt|u, hul unghujulume nppt-

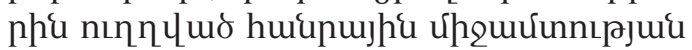

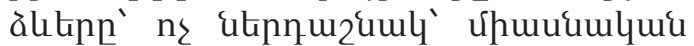

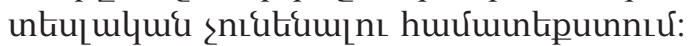

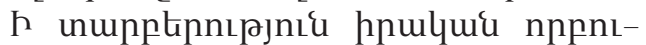

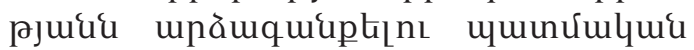

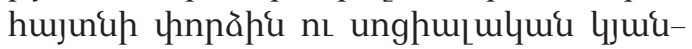
pnıú àlumunnuluó úzulnıرphu, unghu-

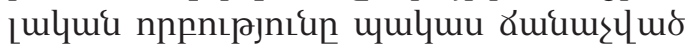
5, n々 lhundtp puguinnluo: hpulque

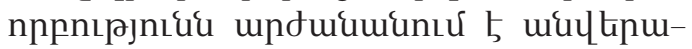

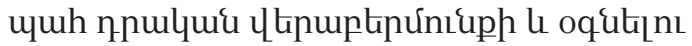

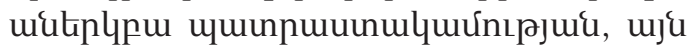

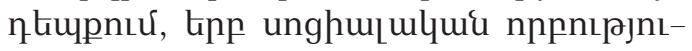

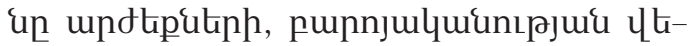
nuptinjul lnu\$thlunnn, punn nt juarnn-

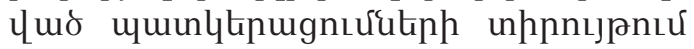

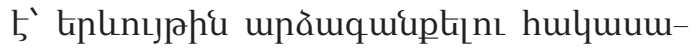

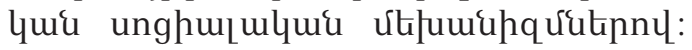

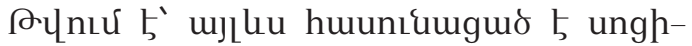

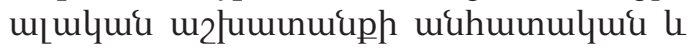
huinujhu úulqunnulh humpuцnnns-

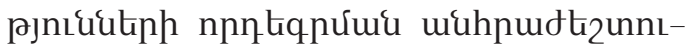

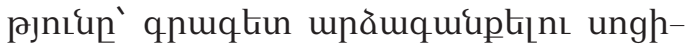

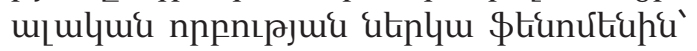

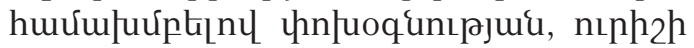

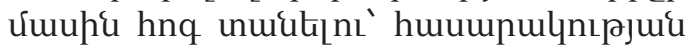

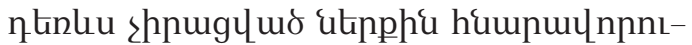

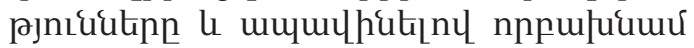

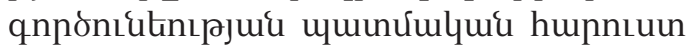

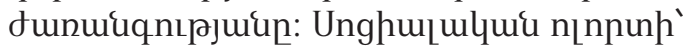
uknlqujnıúu nupugnn pupkihnfunıưutnp le unghu[ulyuu w2fumunnnh huuunh- 


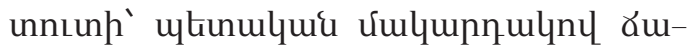

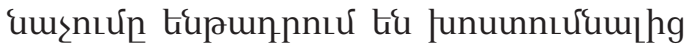

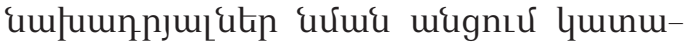

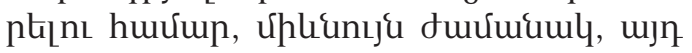
mugnıúu unuiug úmưumqhunulyuu huppulpnıu hujtgulqunqujhí únukgnıưut-

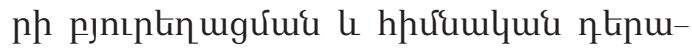
quinunutinh' uhjnınph puntinunutinh nı puntunpulumu quqúultinunıpjnıuutiph

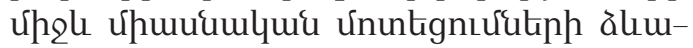

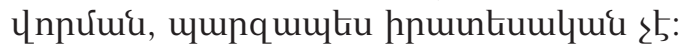

\section{PULUuกrค3กrb}

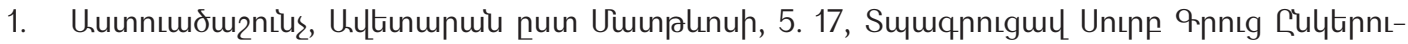
pjniuitiph owfuunun, 1990 р.:

2. Guiunumqhnp, F, 200-215:

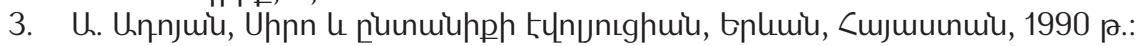

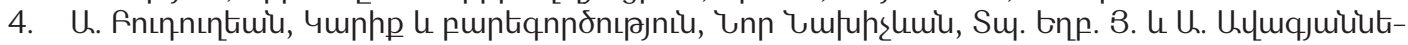
nh, 1901 р.:

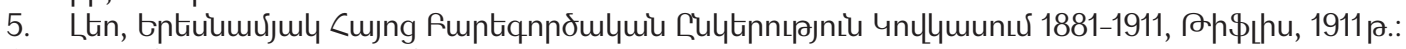

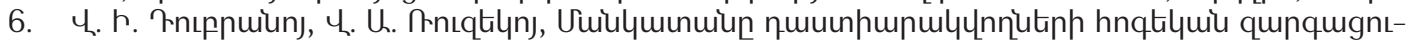
un, Unulyu, 1988 p.:

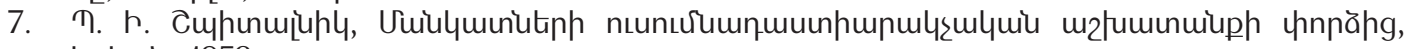
tplumiu, 1953 р.:

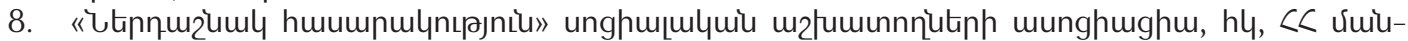

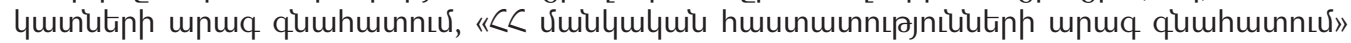

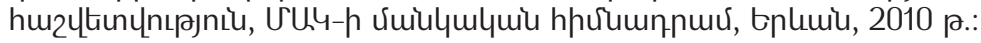

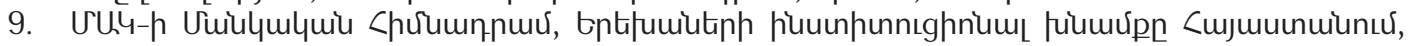
huг2丩tinцnıрлniu, 2010-2012 ра.:

10. www.parsamean.blogspot.com

11. www.hayq.blogspot.com

12. www.krishayas.wordpress.com

13. www.serials.flib.sci.am

14. www.armin.am

15. www.hetq.am

\section{СОЦИАЛЬНОЕ НАСЛЕДИЕ ПО УХОДУ ЗА СИРОТАМИ МИРА АНТОНЯН}

Доцент факультета социологии ЕГУ, кандидат социологических наук

Социальная экспертиза армянского народа по уходу за сиротами в значительной степени лежит в основе эмоционального восприятия и соответстующего подхода к осиротевшим в результате геноцида детям. Анализ социального опыта по уходу за сиротами включает в себя три основных этапа: до геноцида, после геноцида и эпоха советской Армении. Изучая богатое, исторически значимое наследие социального опыта в данный период, статья рассматривает воздействие собенностей социального опыта, характерное для постсоветского времени, на современное понимание ухода за сиротами, присущее социальной работе. В данной статье обосновывается необходимость актуализации государственных и благотворительных подходов к восприятию социальных сирот и детей лишенных семейного ухода. 


\section{SOCIAL HERITAGE OF ORPHAN CARE}

\section{MIRA ANTONYAN}

Associate Professor of Faculty of Sociology, Yerevan State University, PhD in Sociology

Social expertise of the Armenian Nation of the orphan care mostly depends on the emotional perception of genocide orphans struggles and the adequate behaviour towards them. The analysis of social expertise in orphan care includes three main phases: before genocide, after genocide, and during the Soviet era. Examining the rich heritage of social expertise during those phases, the article addresses its effects on modern, post-Soviet perceptions of orphan care in the context of social work. The article justifies the need to modernize state and charity approaches to current perceptions of the problems of social orphanhood, and children deprived of parental care. 